\title{
Design and application of a framework for examining the beliefs and practices of physics teaching assistants
}

\author{
Benjamin T. Spike* \\ Department of Physics, University of California, Berkeley, Berkeley, California 94720, USA \\ Noah D. Finkelstein \\ Department of Physics, University of Colorado, Boulder, Boulder, Colorado 80309, USA
}

(Received 30 November 2014; published 22 February 2016)

\begin{abstract}
[This paper is part of the Focused Collection on Preparing and Supporting University Physics Educators.] We present a newly validated and refined framework, TA-PIVOT (TA Practices In and Views Of Teaching), for examining how physics TAs talk about and how they engage in physics teaching. This work builds upon and extends prior efforts to characterize instructors' beliefs and practices by examining both domains in parallel. We present the comprehensive framework (developed from a study of 31 total TAs) and demonstrate its utility in analyzing both interviews and classroom video observations for a sample of eight TAs. We also discuss how this framework may be used to examine variation in beliefs and practices, track the development of beliefs over time, and inform TA preparation.
\end{abstract}

DOI: 10.1103/PhysRevPhysEducRes.12.010114

\section{INTRODUCTION}

National calls for science education reform have led to widespread changes to physics instruction at the introductory level $[1,2]$. As we modify courses to promote students' active engagement in the learning process and support the development of scientific reasoning [3] and mastery of conceptual physics content [4], we must attend to how instructors in these environments conceptualize and enact their own roles in supporting these goals [5]. One category of educational environments common to introductory physics courses is the smaller, discussion-based sections that are intended to complement lecture and are primarily led by graduate Teaching Assistants (TAs). In recent years these sections have become more student centered through the adoption of research-based curricula, such as the Tutorials in Introductory Physics [6]. Because graduate TAs are partly responsible for establishing classroom norms for student participation in these transformed environments, the instructional beliefs and practices of TAs have become the subject of increasing attention in physics education research [7-9].

We choose to focus on graduate TAs for a number of reasons. First, TAs impact student learning-there is documented correlation between TAs' practices, such as the use of Socratic dialogue, and their students' scores on conceptual postassessments $[9,10]$. Second, as graduate

\footnotetext{
"btspike@ berkeley.edu

Published by the American Physical Society under the terms of the Creative Commons Attribution 3.0 License. Further distribution of this work must maintain attribution to the author(s) and the published article's title, journal citation, and DOI.
}

students, TAs are in the process of reconciling their overlapping roles as both teacher and student. They are being exposed to (and expected to participate in) multiple types of educational environments; these instructional strategies they are expected to support as TAs may be vastly different from those they experienced (and continue to experience) as students. This overlap in roles as student and teacher affords a rich opportunity to examine how TAs make sense of teaching and learning at a critical time in their careers.

Finally, we must also recognize that the graduate TAs of today are the faculty of tomorrow, who will be teaching courses, designing and implementing curricula, and working with future graduate and undergraduate students [11]. In the absence of formal pedagogical training, the primary opportunity for TAs to develop and refine teaching beliefs is through their experiences in the classroom and any accompanying weekly preparation sessions. In many cases, physicists are not required to engage in pedagogical training opportunities after leaving grad school. Therefore, the potential for long-term impact on the beliefs of future faculty encourages us to examine how TA experiences shape the ways in which physicists talk about and carry out their roles as instructors.

Here we build on and extend preliminary work [12] to provide a framework for us as researchers to link the domains of stated beliefs (how TAs talk about instruction) and practices (how they act in instructional environments), which we call TA-PIVOT (Teaching Assistants' Practices In \& Views Of Teaching). In prior work, we demonstrated the potential utility of this framework by applying it to example data without going into significant detail of the coding rubric [13]. In this work, we present the entire framework, describe its development, and give specific examples of how it is applied. 


\section{PRIOR RESEARCH}

\section{A. Nature of instructor beliefs}

The ways in which teachers think about what goes on in the classroom and how they conceptualize their roles in working with students are the subjects of broad attention in education research, spanning preservice $\mathrm{K}-12$ teachers to university faculty [14-19]. Numerous studies have investigated how teachers develop an understanding of the nature of teaching, as well as how such an understanding relates to their instructional practices. As noted by others $[14,15]$, the overall coherence of these studies is inhibited by a general lack of agreement and consistent use of terminology such as beliefs, conceptions, perspectives, attitudes, and values (just to name a few). However, efforts to synthesize prior studies have yielded useful sets of assumptions upon which subsequent studies may be based $[14,16]$. In particular, "beliefs" are generally understood to be tacitly held $[17,18]$, have some connective structure [19], and appear critical to how people define, interpret, and make decisions regarding their behaviors in social settings [16]. In this study, we have chosen to employ the construct of beliefs, and we use it to mean tacit, personally held values and assumptions that shape how teachers interpret classroom situations. In other words, beliefs serve as a lens through which TAs view classroom situations.

In considering instructors' beliefs, we emphasize their contextual nature. Just as student learning of physics must be considered within nested levels of particular contexts [20,21], we must also consider how beliefs are supported, constrained, and shaped by local context. Hoyles [22] describes all beliefs as being situated as a result of being constructed within particular contexts, and Pajares [14] similarly emphasizes the context-specific nature of beliefs. This context dependence will become particularly important in comparing beliefs and practices or considering the "transfer" of beliefs between classroom environments, since different contextual factors may tend to elicit different beliefs.

\section{B. Relationship to practices}

A natural complement to how teachers think about their own teaching is how they enact their roles as teachers in the classroom. Numerous studies have indicated that instructors' beliefs about teaching influence their classroom practices [23], although the exact relationship between beliefs and practices is complex and not a "one-to-one" mapping [24]. This finding underscores the danger of ascribing "enacted belief" labels to classroom practices, as the practitioner and researcher may not agree on the belief reflected by a particular practice.

Furthermore, the relationship between beliefs and practices is mediated by local constraints, and such constraints may weaken the coordination between them [23]. (For clarity, we will use the term coordination when comparing beliefs with practices, and alignment when comparing beliefs or practices with the curricular approach.) One model for this relationship is that instructors' beliefs and practices must be considered as being situated within local context, which mediates the relationship between them. Prior studies have indicated that faculty operating within similar instructional settings negotiate situational constraints differently, and their ability to do so is reflective of the sophistication of their overall teaching model [5].

In investigating the connection between beliefs and practices, we are building upon the approach of recent studies of math and physics TAs that employ a combination of video observations and interviews to provide a more complete picture of their beliefs and practices $[24,25]$.

It is tempting to use teachers' classroom practices as a proxy for their beliefs, and vice versa. However, in the words of Kane et al. [26], considering only teachers' talk amounts to "telling half the story" and limits our ability to investigate the nature and contextual dependence of instructors' pedagogical beliefs. Instead, we must consider both how teachers describe their views of teaching and enact practices in educational settings. In other words, talking to TAs and asking them to describe what matters to them in enacting their role as teachers allows us as researchers to more completely understand the decision-making process that TAs undergo in the classroom.

\section{Development of instruments}

Extensive research has been conducted examining the beliefs and practices of pre- and inservice $\mathrm{K}-12$ teachers and university faculty. To this end, numerous instruments have been designed for documenting instructors' use of particular practices, for example, the Reformed Teaching Observation Protocol (RTOP) [27], Teaching Dimensions Observation Protocol (TDOP) [28], and Classroom Observation Protocol for Undergraduate STEM (COPUS) [29]. Likewise, frameworks exist to characterize instructors' views on the nature of teaching, usually through the use of interviews and surveys [30-32]. Previous research on university academics' teaching has tended to focus on either interview or observational data alone, one as a proxy for the other. In particular, there is not an existing framework that spans the contexts of beliefs and practices in order to examine similar constructs within each domain.

\section{STUDY}

\section{A. Motivation}

Our interest in outlining a framework for bridging beliefs and practices is built upon a number of objectives. First, such a framework would afford greater insight into the nature of beliefs and practices as situated within particular educational environments. Second, it would allow us to uncover and examine instances of consistency and inconsistency between beliefs and practices, and allow us to 
consider the factors contributing to these instances. Additionally, examining the nature of coordination between beliefs and practices allows us to assess the bounds of proxy; that is, we may be able to tell to what extent and under what conditions measured beliefs may be substituted for practices. As noted earlier, a particular tendency in the literature is to ascribe beliefs to particular practices and vice versa; a greater understanding of coordination would in turn streamline future data collection in these settings by providing an empirical basis for the substitution of survey or interview responses for direct observation of practices.

On the more applied side, the creation of such a framework is a critical step in documenting and transforming TA preparation and professional development. There is a recognized need for investigating the effectiveness of existing preparation methods in helping TAs develop and refine productive pedagogical beliefs and practices [33]. Previous research has demonstrated that key factors in the ability of physics faculty to persist in using research-based course transformations include the following: (a) an awareness of course transformations, (b) a belief in the effectiveness of course transformations, and (c) an awareness of barriers or constraints to the implementation of course transformations [34]. Furthermore, instructors' ability to negotiate such barriers appears to be grounded in their broader teaching perspective [5].

The research questions that guided the development of this framework were the following:

(1) Can we design an instrument that is able to distinguish stated beliefs and practices for physics TAs?

(2) Do we observe instances of coordination and/or discoordination between TAs' beliefs and practices?

(3) Can we observe shifts in individual TAs' stated beliefs over the course of a semester?

In this paper, we focus primarily on the first two questions, and will demonstrate the potential of this framework to address the issue of dynamics posed by the third.

\section{B. Methods}

The development of the TA-PIVOT framework originally began with preliminary end-of-semester written surveys designed to elicit TAs' definitions of teaching, as well as their self-reported reflections on how their ideas about teaching changed during the semester. Responses to these surveys tended to be brief, but nonetheless provided emergent themes that informed the design of a subsequent interview protocol.

Drawing from other interview approaches [8,25], we then designed an interview protocol that would allow TAs to elaborate on their responses and, in particular, describe specific teaching instances in greater detail. The interviews (with participant consent) were approximately $40 \mathrm{~min}$ in length and semistructured in that the interviewer could ask to clarify or follow up on specific points at his discretion.
The protocol also included open-ended questions to identify broad themes (e.g., "What is teaching, to you?"), as well as opportunities to reflect on specific classroom events. The complete interview protocol is listed in Appendix A.

In parallel with the interviews, we collected classroom recordings using fixed cameras trained on tables of about four students, in a manner similar to the fixed-camera observational studies conducted by the University of Maryland [35]. Students and TAs were informed beforehand when recordings were taking place, and consent was obtained in accordance with campus human subjects protocols. Researchers were not present in the room during recording. We then identified and extracted video clips in which the TA was present at the table (which we will refer to as "episodes"), which were then coded as described below.

We conducted two rounds of data collection, first with three TAs and then with an additional five TAs in a subsequent semester. In the first (pilot) round, we videotaped TAs during their week 7 tutorial sections and conducted postsemester interviews with each one. In the second (expanded) round, we videotaped five weeks of the TAs' interaction with students (week 4, forces; week 7, work and changes in kinetic energy; week 9, conservation of momentum; week 12, conservation of angular momentum; and week 14, buoyancy). These recordings were conducted using the same fixed-camera methods described previously. Additionally, in order to characterize TAs' beliefs throughout the semester, we conducted pre-, mid-, and postsemester interviews using the same protocol.

The first interviews took place after classes had started, so the TAs already had at least one week of teaching to draw upon. Furthermore, these interviews took place after each of the participants had completed the presemester teaching orientation. Hence, we do not claim that the first interviews reflect the TAs' initial "state" before entering the physics program. It was logistically difficult to arrange interviews with TAs before the orientation, and we were ultimately more interested in examining the ongoing impact of teaching in a transformed learning environment than the effect of a single workshop.

\section{Setting and participants}

Our complete data inventory (spanning multiple semesters) includes 13 TAs who participated in both interviews and videotapings; an additional 18 TAs were interviewed but not videotaped. All of the videotaped TAs served in tutorial sections of the first-semester calculusbased mechanics course (Physics 1) at the University of Colorado (CU), described in detail in Finkelstein and Pollock [36]. TAs in this course lead 1 hour weekly tutorial sections of about 30 students seated in small groups. The TAs facilitate group discussion and engage the students in conversation about their responses and reasoning. 
TABLE I. Study participants, along with their prior teaching experience and incoming familiarity with tutorials. (All names are pseudonyms.) Starred participants were part of the initial pilot study that included limited classroom observation and only postsemester interviews.

\begin{tabular}{lclc}
\hline \hline Participant & Gender & \multicolumn{1}{c}{ Prior teaching experience } & Tutorial familiarity \\
\hline Daniel* & M & TA previous semester, different course & Medium \\
Sarah* & F & TA previous semester, same course & Medium \\
Keith* & M & TA previous semester, different course & Medium \\
Caleb & M & Former middle school mentor and college tutor & Low \\
Darren & M & Former undergrad teaching assistant \& tutor & Low \\
Joanna & F & Former undergrad teaching assistant (in tutorials) & High \\
Terrence & M & Former learning assistant & Low \\
Oliver & $\mathrm{M}$ & None & Low \\
\hline \hline
\end{tabular}

In this paper we focus on 8 first-year TAs teaching Physics 1 tutorials (Table I). Three of these TAs, Sarah, Daniel, and Keith (all names are pseudonyms), were pilot study participants serving their second semester as TAs, and none of them had formal teaching experience before their first experiences as TAs the previous fall. In the previous semester, Sarah had also taught Physics 1 (Mechanics) whereas Daniel and Keith had taught Physics 2 (Electricity and Magnetism), the subsequent course in the series, also using tutorials.

The remaining five TAs were part of the expanded follow-up study and thus participated in additional interviews and videotapings. Darren and Joanna had both served as TAs as undergraduates, with Joanna having taught in a SCALE-UP [37] setting with the UW tutorials. Terrence served as a learning assistant as an undergraduate, although he decided not to participate in the accompanying pedagogy course because was busy preparing for the GRE and writing an undergraduate thesis. Oliver was an international graduate student who originally attended $\mathrm{CU}$ as part of an undergraduate research program, and was then accepted to the graduate program. In addition to serving as a college physics tutor, Caleb had also served as a $\mathrm{K}-8$ reading tutor in a foreign country during college.

Over the course of the study, limited interviews and videotapings were also conducted with TAs in laboratory settings of other algebra-based introductory courses for comparative purposes and to test the reliability of the framework across classroom settings. We also collected supplementary data including interviews and videotape of undergraduate learning assistants who worked alongside TAs in tutorial settings [38], early surveys that informed the development of the interview protocol, and field notes from TA preparation sessions. These additional data informed the study but are not reported on here.

\section{Analysis}

Coding of both interview and video transcripts began with initial open coding to identify recurring themes, patterns of speech, and behaviors [39]. The unit of coding analysis tended to be individual statements in interviews, and turns of talk in observed practices. Codes were assigned until both researchers were satisfied that no emergent themes could be identified in the collected data; the assigned codes were then sorted into broader categories that could be compared against existing theoretical models in the literature. Statements could receive multiple codes if applicable, as will be illustrated in the next section. The final coding scheme constitutes the finest grain size of the framework described below.

We used a coding software package (Nvivo) to analyze both the interview and video data according to the developed rubric (Tables III, IV, and V in Appendix B). Coding was performed by a single researcher. In order to assess reliability of the coding schema before proceeding with coding of the full data set, we recruited another researcher to receive $20 \mathrm{~min}$ of training before coding two 40 min TA interview excerpts. The researchers initially agreed on $83 \%$ of coded statements, which rose to $95 \%$ following a thorough discussion of the rubric and subsequent clarification of codes. A similar process was carried out for video coding: A second researcher was trained in the coding procedure and then given a $3.5 \mathrm{~min}$ segment of video to code. After initial coding, the raters agreed on $78 \%$ of codes. Following discussion and revision, the level of agreement rose to $97 \%$.

\section{RESULTS: FRAMEWORK}

The TA-PIVOT framework has three overall dimensions (agency, goal, and assessment), along with subdimensions for each area (shown in Table II). These dimensions are interconnected and may serve as anchors for an instructor's overall perspective of teaching and learning. Each subdimension is the result of iterative open coding along with consultation of existing literature.

This paper is not meant to serve as an exhaustive case study, but rather as a demonstration of the utility of the framework. In order to introduce the framework and the associated coding schema, we will start by considering representative quotations drawn from TA interviews. We then follow with illustrative vignettes to examine the same TAs' instructional practices, along with summaries of their overall observed practices across a week of episodes (31 
TABLE II. The TA-PIVOT framework, with broad dimensions in bold in the far left column. A more detailed description with the coding rubric is provided in Appendix B.

\begin{tabular}{|c|c|c|c|}
\hline Dimension & Subdimension & Code & Description \\
\hline Agency & Agent & $\begin{array}{l}\text { Teacher } \\
\text { Mixed } \\
\text { Student }\end{array}$ & $\begin{array}{l}\text { Emphasizing teacher direction; teacher tells and students } \\
\text { follow or listen } \\
\text { Emphasizing student contribution } \\
\text { Emphasizing student collaboration and/or collective action }\end{array}$ \\
\hline \multirow[t]{2}{*}{ Goal of instruction } & Knowledge & $\begin{array}{l}\text { Factual } \\
\text { Procedural } \\
\text { Conceptual } \\
\text { Metacognitive }\end{array}$ & $\begin{array}{l}\text { Basic knowledge involving memorization or recall } \\
\text { (things you just need to "know") } \\
\text { Linear, stepwise knowledge to carry out a task or procedure } \\
\text { Knowledge of overarching concepts and connective structure } \\
\text { Strategic, reflective knowledge of own thinking }\end{array}$ \\
\hline & Epistemological stance & $\begin{array}{l}\text { Answer-making } \\
\text { Sense-making }\end{array}$ & $\begin{array}{l}\text { Focus on getting to formal, correct answer } \\
\text { Focus on reasoning and making sense of the answer, } \\
\text { rather than the answer itself. }\end{array}$ \\
\hline \multirow{2}{*}{ Assessment } & Purpose & $\begin{array}{l}\text { Evaluative } \\
\text { Formative }\end{array}$ & $\begin{array}{l}\text { Assessment to evaluate following instruction } \\
\text { Assessment to inform practice or to guide learning }\end{array}$ \\
\hline & Form & $\begin{array}{l}\text { Isolated } \\
\text { Situated }\end{array}$ & $\begin{array}{l}\text { Assessment removed from practice, e.g., giving a test } \\
\text { Assessment within practice, e.g., observing behavior } \\
\text { within social setting }\end{array}$ \\
\hline
\end{tabular}

total videos). In Sec. IV, we will extend this framework to examine the overall consistency of beliefs and practices, highlight variation among an expanded set of 8 TAs, and consider shifts in beliefs over the course of a semester.

\section{A. Agency}

Agency is defined in the literature as the socioculturally mediated capacity to act [40]. In examining agency, we are concerned with who is intended to be active and influential in the learning process. We recognize that in the environment under consideration, both TAs and students are constrained in their possible modes of participation, and thus our definitions of agency are therefore subject to local context. For example, students and TAs are not free to choose the content of the tutorial they work on each week, nor whether or not they use tutorials at all. However, we argue that within this classroom setting there is sufficient latitude for instructors to establish norms for participation through their use of discourse and tools. In doing so, we build on prior work that demonstrates how instructors' implementation of Peer Instruction [41] influences students' perception of classroom norms [5].

Generally speaking, we use this dimension to examine whose contributions are valued and given attention during a classroom interaction. For example, in an interview, one TA stated that his role should be to "step in and tell them how I would do it," which would be coded as teacher agency because it places the clear value on TAs' ideas over the students'. In contrast, a statement that "students should work collaboratively and the TA should not interfere" was coded as student agency because it downplays the TA's role while emphasizing students' collective action. Statements that were coded as mixed involved mutual TA-student action or did not give a clear indication whose contributions were preferred.

In describing her approach to teaching during an interview, Sarah places an emphasis on supporting groups in working together to complete the tutorial. Her role as a teacher is "facilitating [the students'] discussion" and she describes how she is careful not to interrupt an ongoing student discussion. She does note that there are times when the teacher should explain or provide examples, but says that it is best when such ideas are student generated. The following quote reflects Sarah's interest in listening to student ideas:

I think the approach of listening to [the students'] conversation was good because it lets them kind of put out a whole bunch of different ideas, different ways of thinking it, instead of just supposing your way of thinking about it immediately. [Sarah post, 00:10:39]

In contrast, Daniel and Keith's beliefs reflect a strong emphasis on their own agency in a student's learning process. Daniel describes how students should initially work together in struggling through challenging problems, but his role is then to "tell them how I think about it, so they can see how my mind works." Furthermore, Daniel believes it is important for the TA to know the material "cold" because if he needs to stop and think in front of the students, then they will stop paying attention. Daniel describes himself as "not interested" in hearing students" incorrect responses, preferring to step in with a correct explanation: 
I would prefer to see that they got to the wrong track and then get them to the right spot, and then [have] me tell them the way I would think about it, correctly, rather than hear them like, talk about and go around in circles about stuff and try to follow where they were going. I would prefer to just get to the point where the confusion set in and tell them how I did it, and then hear them go, "Ohhh... okay." [Daniel post, 00:32:16]

Similarly to Daniel, Keith states that a primary role of the TA is answering student questions. He expresses surprise that some of the learning assistants he worked with were not always able to answer student questions:

I guess, initially I was expecting [the LAs] to be able to answer all the questions the students had, and then I realized, well, maybe that's not their job, thats the TA's job. [Keith post, 00:37:20]

Figure 1 compares the coding results for each TA's beliefs along the dimension of agency. We present absolute counts of codes for agency during the interview session. Using Fisher's exact test, Sarah's distribution of codes are statistically different from both Daniel's $(p<0.01)$ and Keith's $(p<0.01)$; Daniel and Keith's distributions are not statistically different from one another.

\section{B. Goal of instruction}

Goals and intended outcomes are a widely recognized component of instructor beliefs [42]. This dimension corresponds to the intended outcome of the learning process, and represents a condensed version of the taxonomy of the cognitive domain drawn from Anderson [43]. Within this dimension, we separate instructors' contentbased goals from their epistemological stance toward answer making or sense making [5].

Although the tutorials themselves are conceptually focused, other forms of knowledge are emphasized in various other course components. For instance, weekly homework sets assigned through the CAPA online homework system [44] emphasize traditional physics problem solving with numerical answers and no prompt for

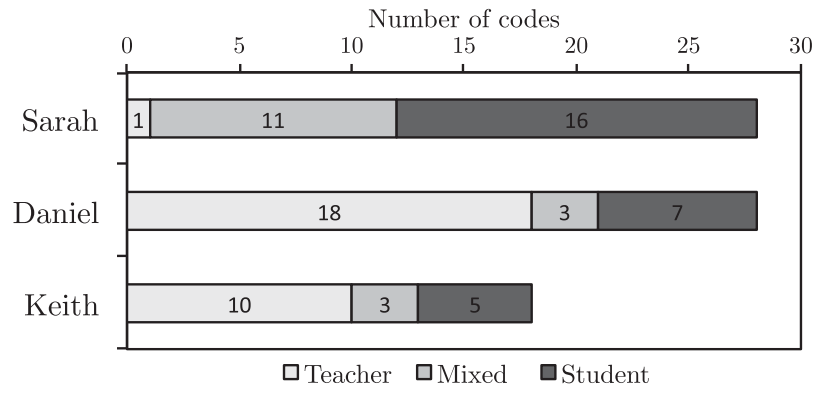

FIG. 1. Coded instances of beliefs along the agency dimension for Sarah, Daniel, and Keith. explanation. TAs may choose to integrate these other cognitive levels within the tutorial setting, or keep them separate.

Keith's goals for his students appear to be a focal component of his beliefs about teaching, as he spent a greater fraction of his interview time than other TAs describing what he wants students to learn about physics. Here is how he responds to a question about what he finds most and least useful about the UW tutorials:

Sometimes I feel like the way [the tutorials] introduce the equations, or prompted the students to write down equations or start doing math was not the best way of doing it... [The students] just weren't in the mode where they were kind of thinking about these physics equations, and it was always hard to get people to recall. You know, I'd be like, "This makes sense because, well, what is momentum equal to, in terms of mass and velocity?" They'd be like, "Ummm," they'd have a hard time thinking about that kind of stuff. I guess I feel like having more problem solving and mathematics kind of informs the intuition and vice versa, it's good to have this kind of back-and-forth way of thinking about it. [Keith post, $00: 23: 42]$

In this quote we see a sampling of the type of understanding Keith wants students to develop. First, there is an explicit focus on factual knowledge, in that he wants students to be able to recall formulas when they need them. In addition, he expresses that activities that focus on problem solving support the development of physical intuition in a "back-and-forth" way. We coded this as a combination of procedural and conceptual knowledge.

Compared with Keith, Daniel spent less time describing the types of physics knowledge he expects students to use and learn in the classroom, but he expressed confidence in what he did say (verbal stress indicated):

For instance, I'll set up a Newton's laws problem where you have forces and I'll say, "This is the way you always do it, you always set up the free-body diagram, then you always write down each component of the Newton's second law, and then once you've got that all written down, the next step is to try and combine it in ways..." [Daniel post, 00:16:35]

Here we see a clear focus on procedural knowledge, but in a more fixed, rote manner indicative of factual knowledge. Effectively, Daniel is emphasizing a process for students to internalize rather than adaptable strategies that students can reconstruct.

Sarah also emphasizes students' problem solving abilities, but discusses it in the context of connecting concepts learned in the tutorial setting to solving the types of traditional physics problems that appear on the weekly homework assignments: 
... just because [students] have to do that for the exams and things, or they just read a problem and they're like, "I know about these concepts, but I have no idea how to connect it to a problem, or what to look for in this problem that tells me what concepts I need." And some of that might be they need to get used to doing that kind of problem and it will come as they take more physics classes. I think that was difficult for some students. [Sarah post, 00:26:47]

In this way, Sarah appears similar to Keith in valuing procedural and conceptual knowledge. Sarah also found ways to reconcile physics concepts with students experiences outside the classroom, such as in this example:

Like when we did pressure this semester, one thing that was helpful was, for understanding that it only depends on depth, is so if you dive down to the bottom of a swimming pool, you know, it really hurts your ears, and it doesn't depend on what the sides of the pool looked like, or how wide the pool is, it's just how deep you go. So that was very helpful for people. [Sarah post, 00:37:12]

Here, using prior physical experience to understand a feature of pressure was coded as valuing sense making.

Figures 2 and 3 show the distribution of knowledge and espistemological stance codes for the Sarah, Daniel, and Keith. Procedural knowledge was the only knowledge subdimension mentioned by all three pilot TAs, mostly having to do with students' abilities to solve traditional

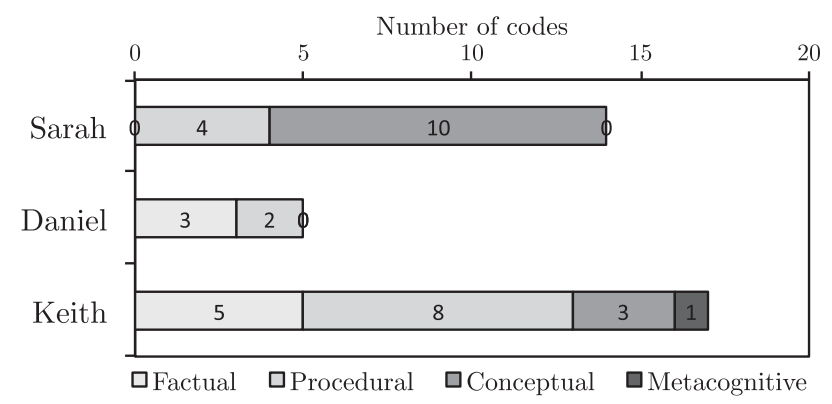

FIG. 2. Coded instances of beliefs along the knowledge dimension.

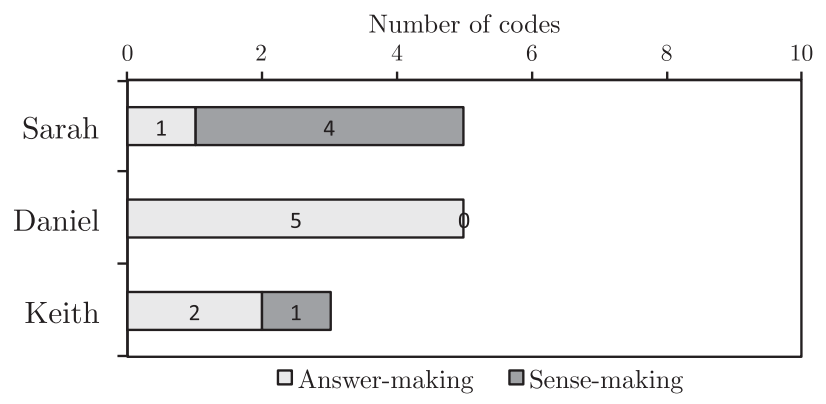

FIG. 3. Coded instances of beliefs along the epistemological stance dimension. computational physics problems. This sentiment was common among many of the interviewed TAs; they generally support the development of students' problem solving skills, although there are differences in opinion on what role the tutorial should play in that process. Keith expresses that there should be more traditional problem solving during the tutorial sections, going as far as to suggest that part of the recitation time be dedicated to showing examples of how to set up and solve mathematical physics problems. Sarah thinks the tutorials serve a role that is complementary to traditional problem solving.

\section{Assessment}

Assessment is a critical component of teaching practice. In this dimension, we examine how an instructor gathers information about a teaching situation, and to what end this information is to be used. For example, an instructor may assess students' understanding by reading what they have written in their tutorial book, by gauging their response to challenge questions, or by observing their manner of participation in a group. The assessment dimension also includes the ways in which TAs use questioning to understand classroom situations, and also encompasses broader perspectives on the role and nature of assessment in instruction.

Our coding subdimensions for the assessment dimension identify particular features of formative and summative assessment, including the intended purpose of assessment and the form it takes relative to instructional practice. In applying the coding rubric, it can be difficult to identify these forms of assessment based on observed practices alone. Instead, we identify features of assessment that can be more readily observed, and these can be mapped onto commonly identified components of formative and summative assessment.

The form subdimension can be somewhat constrained by the TAs'assigned role. For example, in the tutorial environments under consideration, TAs are discouraged from giving quizzes or discussing homework, so we are only able to observe a subset possible practices around assessment. For this reason, we will merge the form and purpose subdimensions of assessment for the subset of TAs presented here.

In this quote, Keith describes a particular way that he assesses student understanding:

[The students] had a hard time understanding what the new words, their meaning in physics class meant, as in understanding that things like "velocity" and "acceleration" were very precise terms that meant something. And that they're supposed to be used in certain contexts. ... they would get confused about speed and velocity because they wouldn't get in the habit of using physics terms. [Keith post, 00:07:34]

To Keith, students' use of informal language is an indicator of a lack of understanding. This is interesting 
framing on Keith's part because he is conducting assessment based on students' forms of participation in the social setting (situated). The language he uses, however, is indicative of a more summative perspective on assessment, as he does not describe how he might build upon students' everyday vocabulary to help them move toward using physics terminology. This is coded as evaluative assessment, rather than formative.

Sarah also describes how she uses questioning in particular ways:

Or also, sometimes we came up with interesting questions to ask people who finished early, which was kind of fun. Or questions you can ask to try to deepen intuition of, or gauge their understanding of it without just asking the answer to the question in the tutorial. [Sarah post, 00:40:56]

In Sarah's view, questioning can serve as a means to "gauge understanding" (evaluative) but also to "deepen intuition" (formative). Likewise, Sarah also described in her interview how part of her role is "asking questions to answer questions," which also reflects her belief in using questions to drive discussion in addition to evaluating student understanding.

A summary of assessment coding is shown in Fig. 4. Compared to other teaching components, the TAs appear less explicit in describing their beliefs about assessment in the classroom. In the absence of ongoing pedagogical training, these TAs appear less accustomed to terms (and concepts) such as "formative" or "summative" in describing their own assessment practices. In fact, these terms were not mentioned in interviews with any of the 13 TAs who were videotaped. We note that Sarah tends the most toward emphasizing more formative types of assessment, although both the amount and variation are not as great as in other dimensions.

By looking across these dimensions holistically, we can paint a broader picture and infer a more recognizable (if not stable) model of the TAs' teaching philosophies. We described previously how the relative focus of each TA on these dimensions provides insight as to their overall models of teaching. For instance, Keith's focus on content

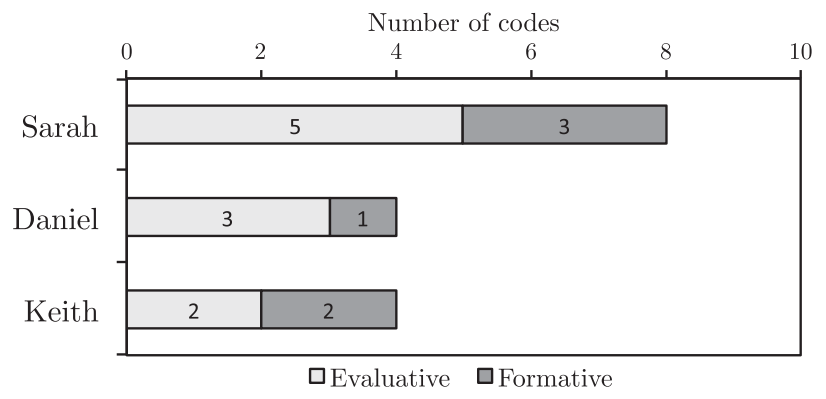

FIG. 4. Coded instances of beliefs along the assessment dimension. goals (using interview talk time and number of codes as an indicator) are reminiscent of the teaching model that Kuhs and Ball [45] describe as content focused with an emphasis on performance. In this model, student mastery of mathematical rules and procedures is emphasized, with the instructor serving primarily to demonstrate and model problem solving tools and methods.

While we do not argue here for a robust and consistent model of beliefs, we can use these dimensions to more carefully examine TA practices, thereby allowing us to consider TAs' described and enacted models of teaching side by side. In the next section, we examine TA practices through the lens of the TA-PIVOT framework.

\section{Application to practices}

To illustrate the application of the TA-PIVOT framework to TAs' instructional practices, we use it to analyze several classroom episodes. In practice it is useful to examine complete episodes of TA practice and then unpack the observed practices in that episode across each of the three dimensions. We first provide examples of how this is done, and then present the coding results for TA practices.

We begin by considering one particular topic area covered by each of the TAs during week 7 of Physics 1 , after which we will summarize the video coding results across all observed episodes captured from videotape data during that week. The following vignettes are drawn from the "Work and changes in kinetic energy" tutorial [6]. In this tutorial, students are presented with a scenario in which a force $F_{0}$ is applied to two blocks, $C$ and $D\left(m_{C}<m_{D}\right)$, as they move between two lines on a frictionless table. In the question under consideration, students are asked to agree or disagree with a student who claims that block $C$ will have a greater kinetic energy than block $D$ at the time they each cross the finish line. On the next page, the tutorial prompts the students to check the consistency of their reasoning with the work-energy theorem.

We find this question particularly interesting to focus on because it provides a classic example of the UW tutorial "elicit-confront-resolve" model, in which students are presented with a question that is known to commonly elicit incorrect ideas; then prompted to consider an alternative explanation; and finally guided toward resolving the inconsistency [46]. Very often these types of questions present a dilemma for TAs to "troubleshoot" student reasoning on the spot or allow students room to persist with incorrect ideas knowing they will be prompted to resolve it on their own.

\section{Daniel}

1 [Daniel sits down at a table where S1, S2 are 2 seated.]

3 Daniel: Do you guys feel good about the one 4 on the previous page, this guy? You agree!

5 Uh oh. 
6 S1: Yeah, see, I didn't want to agree.

7 D: So, let's think about this. So, we know

8 that the force is the same on each block,

9 right?

10 S1: Yeah.

$11 \mathrm{D}$ : And we know the distance the blocks

12 travel is the same.

13 S1: Right.

14 D: So, what's work? Force times distance.

15 And work is equal to change in kinetic energy.

16 S1: Right.

$17 \mathrm{D}$ : So if the force is the same on both and

18 the distance traveled is the same on both, the

19 work done on both-

$20 \mathrm{~S} 1$ : Is the same.

$21 \mathrm{D}$ :- is the same. Which means the change

22 in kinetic energy of both is the same.

$23 \mathrm{~S} 2$ : But if you look at it, like $F=m a$,

24 then wouldn't the acceleration of one have

25 to be bigger than... delta KE, 'cause like

$261 / 2 m v^{2} \ldots$

27 D: It' s not totally obvious how you would

28 relate the acceleration from, to the veloc-

29 ity. But you can apply this principle that

30 the change in kinetic energy is equal to work

31 done.

32 [S1, S2 erase their previous responses and re-

33 spond again. Daniel waits a few moments and

34 leaves.]

We notice immediately that this interaction is very brief, and that almost every "correct" or scientifically accepted contribution to the discussion comes from Daniel. Note that upon observing that the students had responded incorrectly to the first question, he chooses to explain in detail his own reasoning instead of asking them to explain how they reached their conclusion. Throughout this conversation, Daniel provides few opportunities for the students to express their own ideas, apart from agreeing or disagreeing with his explanation. In line 14, he even answers his own question. Furthermore, when S2 raises a salient point about the relative motion of the two blocks in line 23, Daniel does not address the content of the question. We code each of these practices as emphasizing teacher agency.

Daniel's goals in this episode focus on the expressions for work and the work-energy theorem (factual), along with demonstrating the logical progression (conceptual). With regard to epistemology, this interaction is geared toward "fixing" the students' incorrect responses (answer making). Finally, although Daniel asks no questions during this episode, he initially uses the students' written responses (isolated) to assess their understanding (evaluative).

It's worth noting that this interaction is almost identical to one from another of Daniel's sections during the same week:

1 [Daniel approaches table.]

2 S1: Can we like, check this with you-
3 Daniel: Yes.

4 S1:- like, that that's right?

5 D: You say-

6 S1: We said that we agreed.

7 D: Right.

8 [TA pulls up a stool and sits down]

9 D: Uhh, sooo...

10 D: On these two blocks, we know there is the

11 same force. [Points at diagram in S1's book]

12 S1: Yeah. [Nods emphatically]

13 D: Right? And we know these two blocks

14 travel the same distance.

15 [S1 nods]

16 D: So that means that the work done on the

17 two blocks is exactly the same. And if the

18 work done on the two blocks is exactly the

19 same, it means that their changes in kinetic

20 energies are exactly the same.

21 S1: Ohhhhh.

22 D: So if the changes in kinetic energies are

23 exactly the same, then the student's wrong.

24 [S1 and others at table erase their previous

25 response]

In particular, Daniel's step-by-step explanation from lines $10-23$ is reused almost verbatim from the previous example, and is triggered by the same observation that the students agreed with the incorrect student statement from the tutorial. This pair of episodes illustrate the type of "scripted" interactions that can be common for instructors, even in transformed environments. In this case, Daniel appears to have a practiced explanation for this question that he applies upon observing incorrect reasoning.

\section{Sarah}

We previously observed that Sarah's stated beliefs were different than Daniel and Keith's, so we can examine her interactions with students to see if these differences hold up in practice.

1 Sarah: So what did you think about the ki-

2 netic energies of the two [blocks]?

3 S1: They're different, but we can't tell which

4 one's greater and which one's less because we

5 don't know how much... [moves hands alter-

6 nately up and down]

7 S: So how do you know they're different?

8 S1: They both have different masses and dif-

9 ferent velocities.

$10 \mathrm{~S}$ : Okay, but what do we know about the work 11 done?

12 S1: The same.

$13 \mathrm{~S}$ : And we had this work-kinetic energy the-

14 orem. So if we just look at that, it seems like 15 the kinetic energies ought to be the same.

16 S4: The change in kinetic energy should be 17 the same. 
18 S: Right. So if they start at zero velocity...

19 [S1 erases a previous response.]

20 S3: And it's zero kinetic energy.

21 S4: So the final kinetic energy should be the

22 same.

$23 \mathrm{~S}$ : Right. So is that consistent with what we

24 were talking about? We don't really know a

25 whole lot about... I guess you could work it

26 out carefully, but it's sort of not very easy to

27 see.

28 S4: Yeah. Yeah.

29 S: And that's why we do this work-kinetic en-

30 ergy thing, is because it makes a really simple

31 way to solve some problems.

32 S4: Okay. So the kinetic energies are the

33 same. [To Sarah] Right?

34 S: Right.

35 S4: Because the net work is the same, the

36 change in kinetic energies are the same.

$37 \mathrm{~S}$ : And what you guys were thinking about

38 was right, it's just it's hard without knowing

39 specifics to say exactly what the velocity and

40 mass relationship is, but it should work out

41 to be this exact same answer. Looks good.

42 [Sarah leaves the table.]

This episode illustrates a few features of Sarah's teaching. First, she gives explicit attention to student reasoning (line 7), prompts for reflection (lines 23-24), and validates student thinking (lines 37-38). Each of these practices is aligned with an emphasis on student agency. However, the overall interaction is centered around guiding students toward a particular result, in a similar manner to Daniel in the preceding excerpts. Sarah also alludes to problem solving (lines 29-31) (procedural knowledge), and selfconsistency (lines 23-24) (metacognitive). Furthermore, we coded her bypass of the kinematic approach (lines 38$41)$ as aligned with answer making.

There is another pattern of interaction Sarah tends to follow, which is a "checking" mode in which she goes over a page of the tutorial, as in this episode:

1 Sarah: Do you want to [go over the third

2 page] real quick?

3 S2: Yeah.

4 S: OK. So did you go over page 42 ?

5 S2: We didn't. Do you want to go over that

6 really quick too?

$7 \mathrm{~S}$ : Cool. Alright, so in that one, what's the

8 work done on [block] A?

9 S2: Positive.

$10 \mathrm{~S}$ : OK. And then on B.

11 S2: Positive.

$12 \mathrm{~S}: \mathrm{OK}$, so then if I define the net work to be

13 the sum of those two, that has to be... what?

14 S2: Just A plus B?

$15 \mathrm{~S}$ : OK, yeah. So it's like some positive non
16 zero number. So-but what's the net force

17 on the system?

18 S2: Zero.

19 S: So, how is that consistent? There seems

20 to be some reason that I can't calculate the

21 net work from the net force. So what would

22 that be?

23 S3: Can you ask that question again?

24 S: Yeah. So it looks like if I tried calculating

25 net work from a net force that equals zero, I

26 kind of expect-

27 S2: The net work would equal zero?

$28 \mathrm{~S}$ : So there's some reason that the net force

29 doesn't tell me about the net work.

30 S2: Because you have to consider the objects

31 independently?

32 S: Yeah, and so we know, force has a direc-

33 tion, right?

34 [S2 nods]

35 S: Does work have a direction?

36 S2: No. In the sense that it matters which di37 rection the force and displacement are going,

38 but not-

39 S: Right, but it doesn't have its own direc-

40 tion. Like, you can't say work is pointing at 4145 degrees-

42 S2: Right.

$43 \mathrm{~S}$ : OK. So, work is kind of a scalar. So if

44 you add two scalar quantities, as long as they

45 have the same sign, they're not gonna cancel

46 out. But for a vector, the direction matters,

47 right?

48 S2: Right.

49 S: So if I add two forces, then they can cancel.

50 But the work doesn't necessarily-so that's

51 why you consider them independently.

We observe in this episode that Sarah is asking primarily short, recall-based questions focusing on procedural and conceptual knowledge, with an occasional check for consistency (metacognitive). Furthermore, although three students are seated at the table, she engages almost exclusively with S2. The differing nature of these interactions results in the more mixed approach to agency in her overall classroom practices.

\section{Keith}

TAs sometimes make their content goals immediately apparent through their classroom practices. In this episode, Keith interacts with a group who has completed the tutorial about 10 min early:

1 Keith: You guys are done?

2 S2: Yeah.

$3 \mathrm{~K}$ : Totally done?

4 S3: Done with what?

5 S2: We're speedy physics doers. 
$6 \mathrm{~K}$ : Alright. Then you get the bonus-the

7 bonus tutorial.

8 S2: Can we just do CAPA?

$9 \mathrm{~K}$ : You can just do CAPA. This will take-

10 this will only take a couple of minutes.

11 S3: We only have a couple of minutes.

12 LA: You still have ten minutes to go.

$13 \mathrm{~K}$ : You still have ten minutes.

14 S2: Yeah, that's why we're going to do

15 CAPA.

$16 \mathrm{~K}$ : This will take up a few minutes. Ready?

17 OK. If I told you this, would you believe me:

18 kinetic energy-

19 S3: No. (laughs)

$20 \mathrm{~K}$ :-final is equal to kinetic energy initial

21 plus delta kinetic energy.

22 S2: Initial plus...

$23 \mathrm{~K}$ : Initial plus the change is equal to final,

24 right?

$25 \mathrm{~K}$ : Because final minus initial-

26 S2: Right. Right.

$27 \mathrm{~K}$ :-is equal to the change. So I just... OK?

28 S2: Yup.

$29 \mathrm{~K}$ : Delta KE, what do you know it is from

30 this tutorial?

31 S2: Uh, work.

$32 \mathrm{~K}$ : OK, so, kinetic energy final, how can we

33 write that in terms of $m$ and $v$ ?

34 S2: In terms of what?

$35 \mathrm{~K}: m-$

$36 \mathrm{~S} 2: 1 / 2 m v^{2}$.

$37 \mathrm{~K}$ : Yeah. $1 / 2 m v$ final squared. Same thing

38 for initial. 1/2 $m v$ initial squared. Plus work.

$39 \mathrm{~K}$ : What's work in terms of force and dis-

40 tance?

41 S2: Force times distance. Force times dis-

42 placement.

$43 \mathrm{~K}$ : Force times distance. We're talking in one 44 dimension, so let's just do force times dis-

45 tance, pretend that the cosine theta... So the

46 work becomes "plus force times distance."

$47 \mathrm{~K}$ : What's force?

48 S2: Mass times acceleration.

$49 \mathrm{~K}$ : Does this equation look familiar?

50 S4: Yes it does.

$51 \mathrm{~K}$ : What if we were to cancel out the

$52 \mathrm{~m}$ 's... and multiply everything by 2 ?

53 S2: $v$ final equals $v$ initial plus $2 a d$. Look at 54 that! What?

$55 \mathrm{~K}$ : So you don't need to remember this now,

56 because you know this. So you can always go

57 from here to here.

58 S2: Cool.

59 K: First equation, and then just... So you

60 need one, two, three, four equations. And
61 you can get from here to here.

62 S2: Cool.

$63 \mathrm{~K}$ : But you know all these things, these are

64 really simple, you can remember them all.

$65 \mathrm{~K}$ : That's the bonus lecture. So you're prob-

66 ably wondering why doesn't this have time in

67 it, where the heck does it come from when we

68 first learned it, it comes from kinetic energy.

69 S2: Sweet.

What we observe in this episode is basically an extended lecture on Keith's part, with a clear focus on mathematical derivation. Although it is not immediately apparent from the transcript, the screenshot in Fig. 5 illustrates how Keith is writing out each line of the proof while the students watch (teacher agency). Keith is demonstrating a proof of an earlier kinematic equation using newly introduced workenergy principles (procedural and conceptual knowledge). Keith provides frequent prompts for student contributions (lines 29, 32, 39, 47, and 49), but they are all closed-form questioning serving to fill in the blanks along his predetermined path (answer making). In line 55, Keith gives his motivation for showing the students this proof, which is to use the more general formula instead of the old one (factual and procedural knowledge). Keith's students do not seem to share his enthusiasm for this proof; toward the end, S2, who was the most persistent about moving on to the CAPA homework, makes repeated attempts (Lines 58, 62, and 69) to disengage from the conversation.

These vignettes represent a subset of the episodes coded for the "work and changes in kinetic energy" tutorial. Each of these episodes can be coded using the TA-PIVOT rubric, and we do so in order to observe overall patterns. Although we examine the full set of practices coding results in the next section, we will first comment that the TAs tend to emphasize teacher agency in the classroom-in particular Sarah, who on average spends the longest time at each table. Although she asks questions frequently and allows ample response time for students, Sarah also dedicates a large portion of time to directly explaining or clarifying

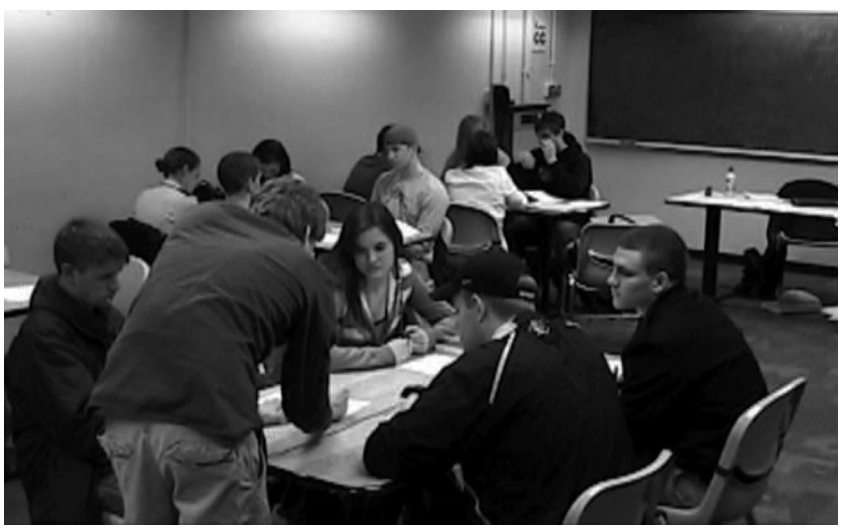

FIG. 5. Keith demonstrating a proof. 
ideas to students. In once instance, a group interrupts her in the middle of an explanation to say, "We think we get it."

With respect to the TAs' goals, there is a clear emphasis on factual, recall-based questioning among the TAs. In fact, Daniel relies exclusively on testing students' factual knowledge during week 7, whereas Sarah and Keith demonstrate an additional but relatively more limited focus on conceptual and procedural understanding. This is somewhat surprising due to the conceptual focus of the tutorials, and encourages us to think about the TAs' reliance on this lower-order cognitive domain. Additionally, Sarah's practices tend to emphasize students' ability to interpret and make sense of physics content, whereas Keith's practices focus on the correct, scientifically accepted answer.

We also observe clear differences among the TAs' preferred forms of assessment. Daniel relies exclusively on student responses to his checking-style questions, as well as their written answers, to gauge understanding. Sarah and Keith are more mixed in attending to students' forms of participation in the class, as well as direct questioning, to inform their practice. In the case of Daniel and Keith, this questioning tends to take a more closed initiate-response-evaluate form [47] that constrains student responses along a predetermined path. Although Sarah also engages in this style of questioning, we also observe her using questions to open the discussion further in a more formative manner.

\section{RESULTS: COORDINATION AND DYNAMICS}

\section{A. Comparing beliefs and practices}

In order to compare beliefs and practices coding side by side, in Fig. 6 we present coding results for all eight TA participants. Beliefs coding results are drawn from postsemester interviews, whereas practices codes are aggregated from all observed episodes. Here we use alignment with the tutorial approach as a rough measure of cognitive demand in ways that reflect both our view of the tutorials themselves, and also how they are incorporated in our course setting. We should also stress that we differentiate coordination of beliefs and practices from inflexibility. We view sophisticated teacher knowledge as inherently fluid and adaptive, and we therefore do not associate "sophistication" with any particular component of these dimensions. Rather, we expect more sophisticated instructional practices to be flexibly applied and informed by local contexts and student feedback.

Daniel and Keith's observed practices related to agency appear relatively well aligned with their stated beliefs, both of which emphasize teacher agency. However, they are inconsistent with the intended approach of the tutorial, which is to allow students the opportunity to externalize their own reasoning and resolve disagreements and inconsistencies amongst themselves. In comparison, Sarah is aligned with the tutorials in her stated beliefs but not as well in her practices.
Examples like Sarah's illustrate how challenging it can be to consider and apply pedagogical intentions "in the moment."

Compared to the interview setting, all of the TAs had a greater tendency to emphasize Factual knowledge in the classroom. We also observe indications of consistency between beliefs and practices in TAs' epistemological stances. For instance, Daniel was coded as only answer making in the interview setting, and he had a relatively higher fraction of this code in practice; the same was true for Sarah along sense making. In this instance, Sarah is coordinated with the curriculum in both her beliefs and practices, which may indicate a productive foothold that could be used to help her align other aspects of her instructional practices with her beliefs.

Our earlier observation regarding the TAs' lack of focus on assessment in their beliefs appears to carry over to their practices as well, where we see that questioning is not a focal component of TA practices. When it is, it is primarily used to gauge student understanding rather than allow an opportunity to expand the discussion. This result is surprising to us, given the critical role of assessmentparticularly formative assessment-in instruction. This finding motivates us to reflect on current TA preparation methods and consider how discussions of the assessment accompanied by efforts to incorporate them into ongoing practice would be productive in increasing TA attention to beliefs and practices surrounding these constructs.

Furthermore, we find that TAs in these environments may exhibit surface-level support or buy in to transformed curricula while exhibiting beliefs and practices that appear contrary to the pedagogical intent of that curriculum. Daniel is one example of a self-described supporter of the UW tutorials, having used them as an undergraduate physics student in the same environment that he is now a TA. This observation is consistent with Dancy and Henderson's finding that physics faculty who self ascribe as users of a research-based curriculum may not necessarily implement them as designed [48], and when faculty modify researchbased curricula, it tends to be in a way that emphasizes more traditional forms of teaching [49]. Opportunities to teach using transformed curricula afford a chance for graduate teachers to become deeply engaged with the aims of research-based instructional strategies that will serve them in future teaching; however, as Daniel's example demonstrates, this engagement does not happen on its own.

We notice that the TAs' "centers of gravity" appear to be toward the left side of the chart, indicating a general tendency toward antialignment with the curricular approach. Looking across the dimensions, the TAs' practices appear to be "stratified" relative to one another. That is, the TAs' relative positions along each dimension appear to be coupled (for instance, compare Darren's points with Caleb's). Furthermore, the TAs appeared more mixed around the constructs of agency and knowledge than the other dimensions. Darren in particular demonstrated a consistently teacher-centered approach to his instruction, 
(a) Post-semester Beliefs

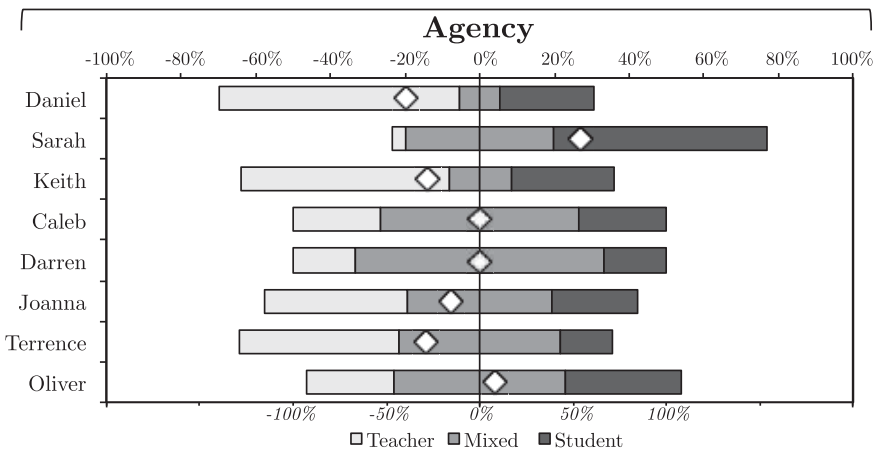

Knowledge

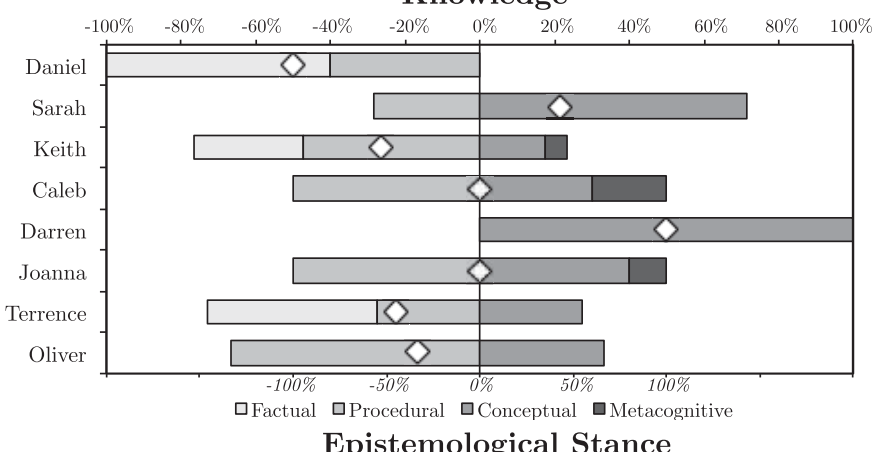

Epistemological Stance

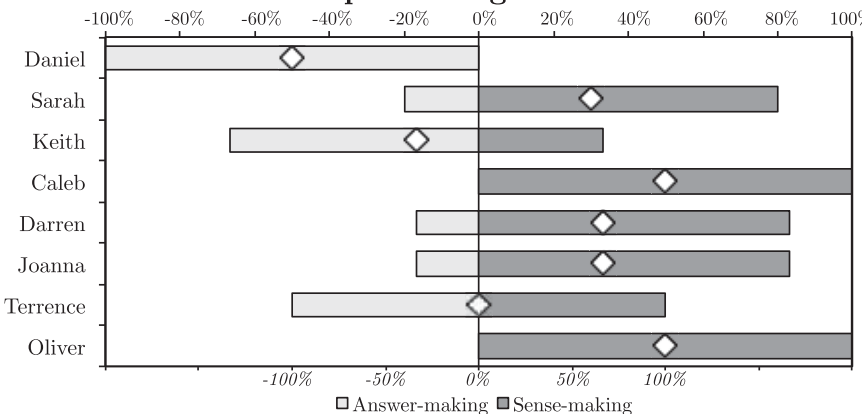

Assessment

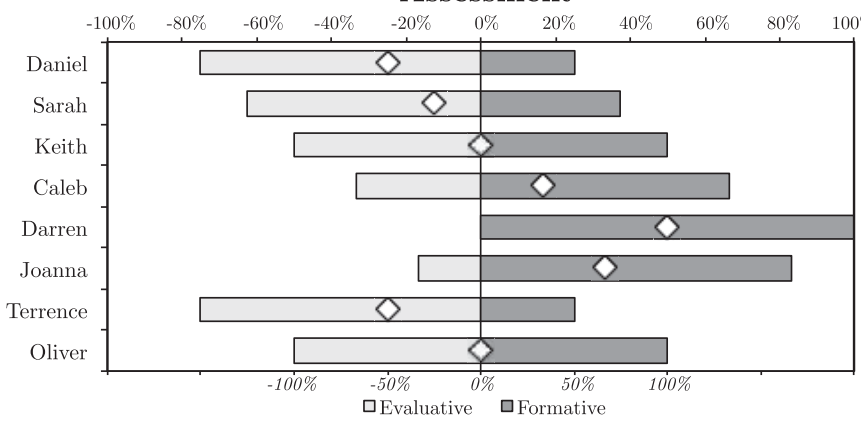

(b) Practices

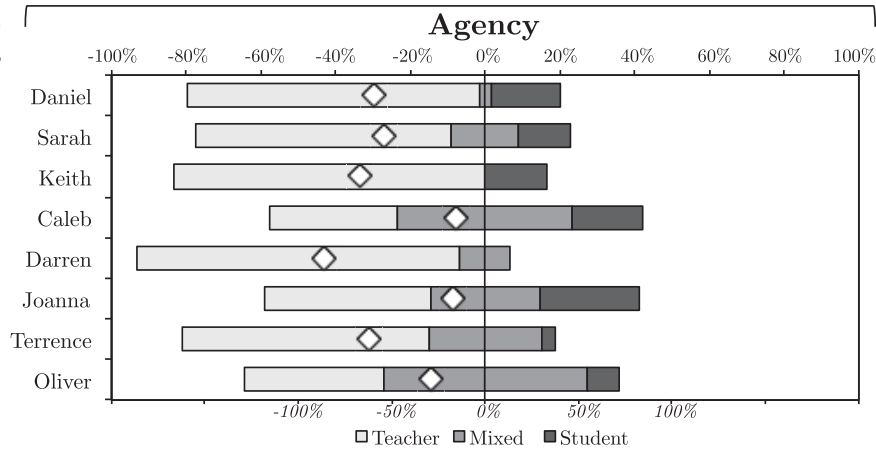

Knowledge

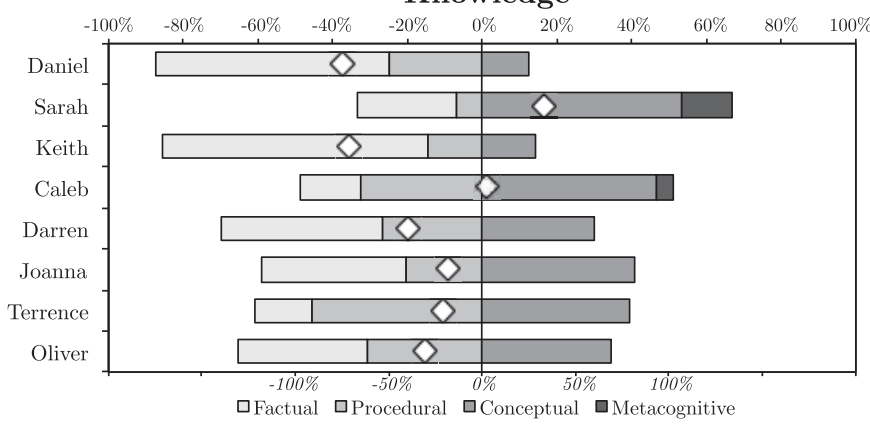

Epistemological Stance

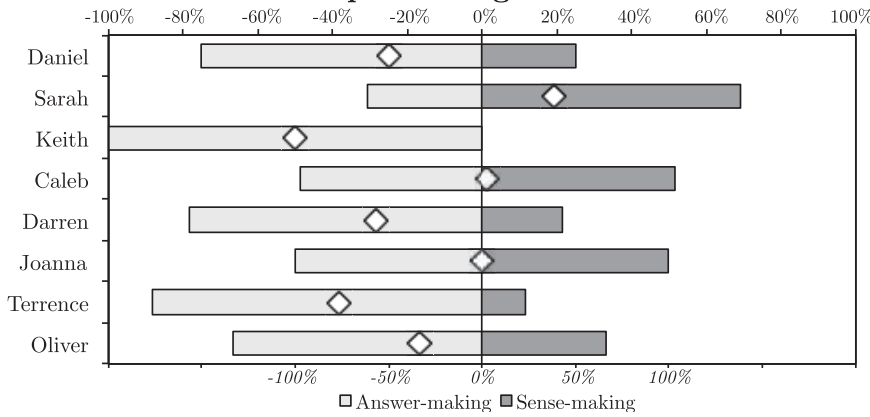

Assessment

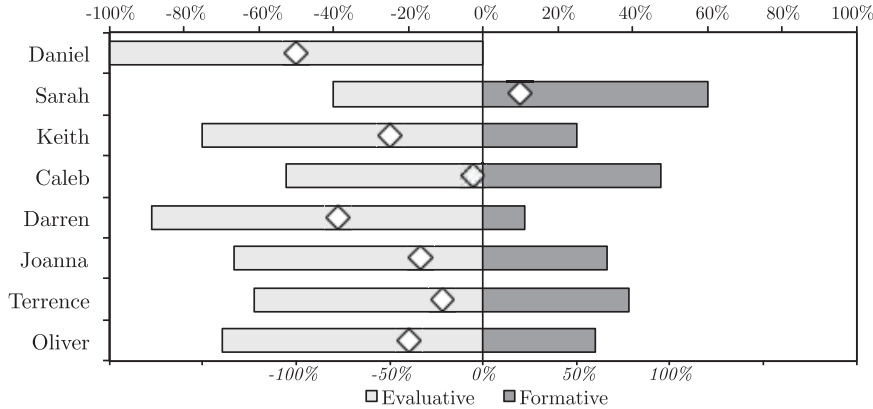

FIG. 6. Side-by-side comparison of coding results for (a) beliefs and (b) practices for all 8 TAs. Percentages along the top indicate percentage of assigned codes for each TA. Percentages along bottom (in italics) correspond to the position of the diamond markers, indicating each TA's median code and overall degree of curricular alignment. Positive values indicate alignment with the tutorial approach, whereas negative indicates antialignment.

primarily characterized by his direct answers to student questions with relatively infrequent prompts for student collaboration or discussion. Although no TA was consistently student centered in his or her approach, Caleb and Joanna's demonstrated the widest range of agency practice codes.

\section{B. Coordination between beliefs and practices}

As illustrated in Fig. 6, we observed instances of TAs whose stated beliefs were aligned with curricular goals (but not aligned with their practices), and TAs whose stated beliefs aligned with their practices (but not the curricular goals). In order to better visualize the degree of 
coordination between TA beliefs and practices, we can translate the Fig. 6 data into a two-dimensional format, as shown in Fig. 7. In this figure we have plotted aggregate coded practices against post-semester coded beliefs. The coordinates of each marker correspond to the positions of the diamonds in Fig. 6.

In this representation, a TA's location on the chart indicates the degree of coordination between his or her beliefs and practices. Points along the diagonal indicate coordination between stated beliefs and enacted practices, with points further from the origin being more aligned with the curriculum. Points that are above the diagonal indicate that a TA's stated beliefs are more aligned with the curriculum than his or her practices, and vice versa.

A few trends are immediately apparent from Fig. 7. Overall, there is a general trend away from alignment with the curricular approach (i.e., toward teacher agency, answer making, and evaluative assessment). We note that Darren tended to have the largest differences between coded beliefs and practices. On the other hand, Terrence demonstrated a relatively small difference between coded beliefs and practices, although each of these tended to be antialigned with the tutorial approach. We may therefore wonder whether TAs such as Darren are aware of the difference between their described and enacted practices, and whether they would recognize a disconnect upon viewing excerpts of their own teaching.

\section{Dynamics in beliefs}

As many TAs are first-time teachers, we are interested in what impact these teaching experiences (particularly in transformed environments), may have on their stated beliefs about teaching. In Fig. 8 we present both preand postsemester interview coding results using the same two-dimensional representation to illustrate pre-post semester shifts in the TAs' coded beliefs. Only the five follow-up TAs are included in this figure, as presemester interviews were not conducted with the pilot TAs. In this representation, the $x-y$ position indicates the pre-post shift (if any) in the TAs' coded beliefs. Points along the diagonal are indicative of no pre-post shift in beliefs, whereas points above or below the diagonal indicate shifts that are either aligned or antialigned with the curricular approach. Furthermore, the distance between the point and the diagonal indicates the relative size of the shift.

For the most part, although they expressed differing beliefs from one another in the interview setting, the TAs
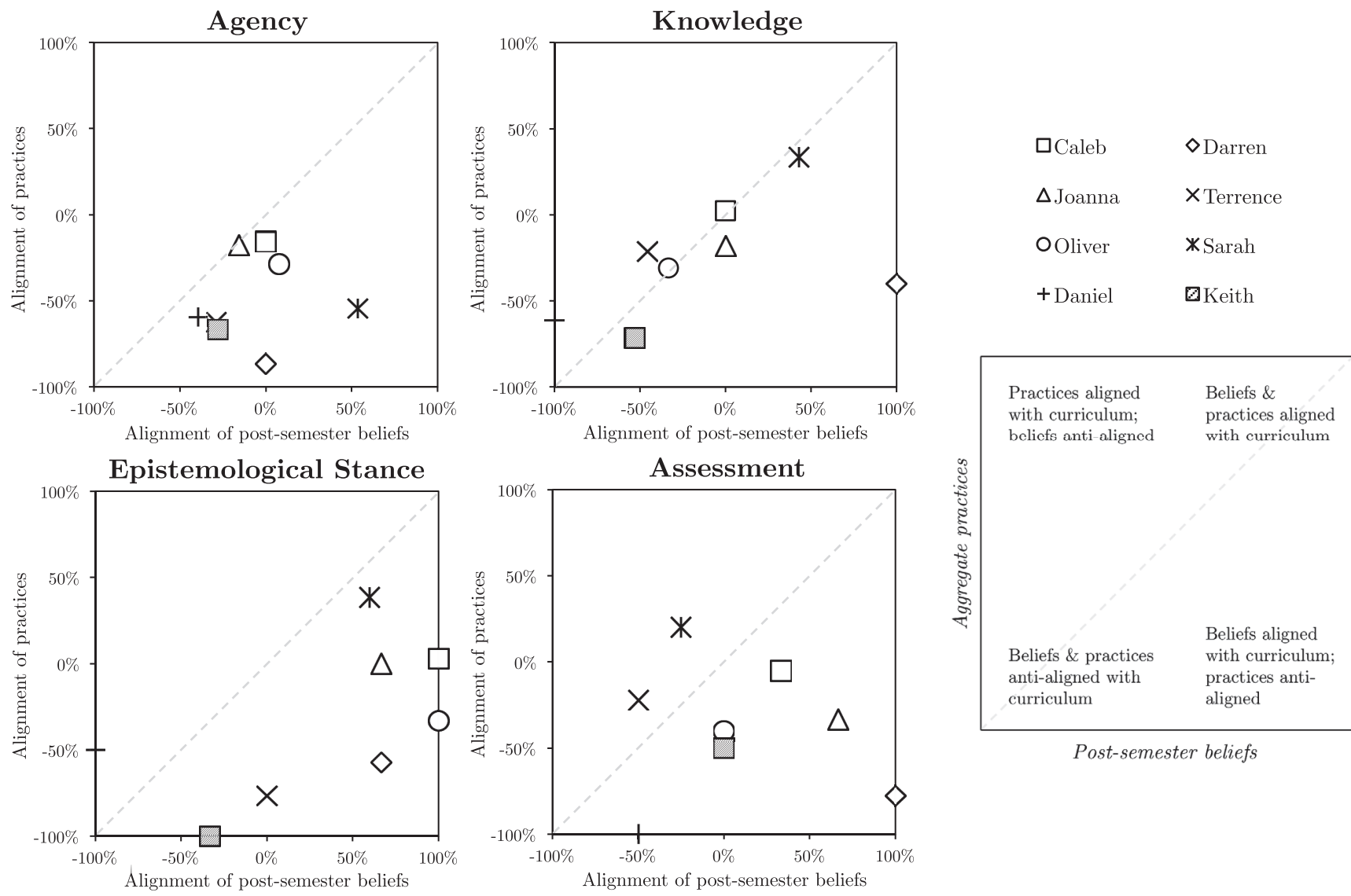

belicfs anti-aligned with curriculum

FIG. 7. Two-dimensional representation of coordination between TA beliefs and practices. For each coding dimension, the marker position indicates the degree of alignment of post-semester beliefs (horizontal) and aggregate practices (vertical). Pilot study TAs have been included for comparison. In this representation, points on the diagonal represent well-coordinated beliefs and practices. (See guide at right.) 

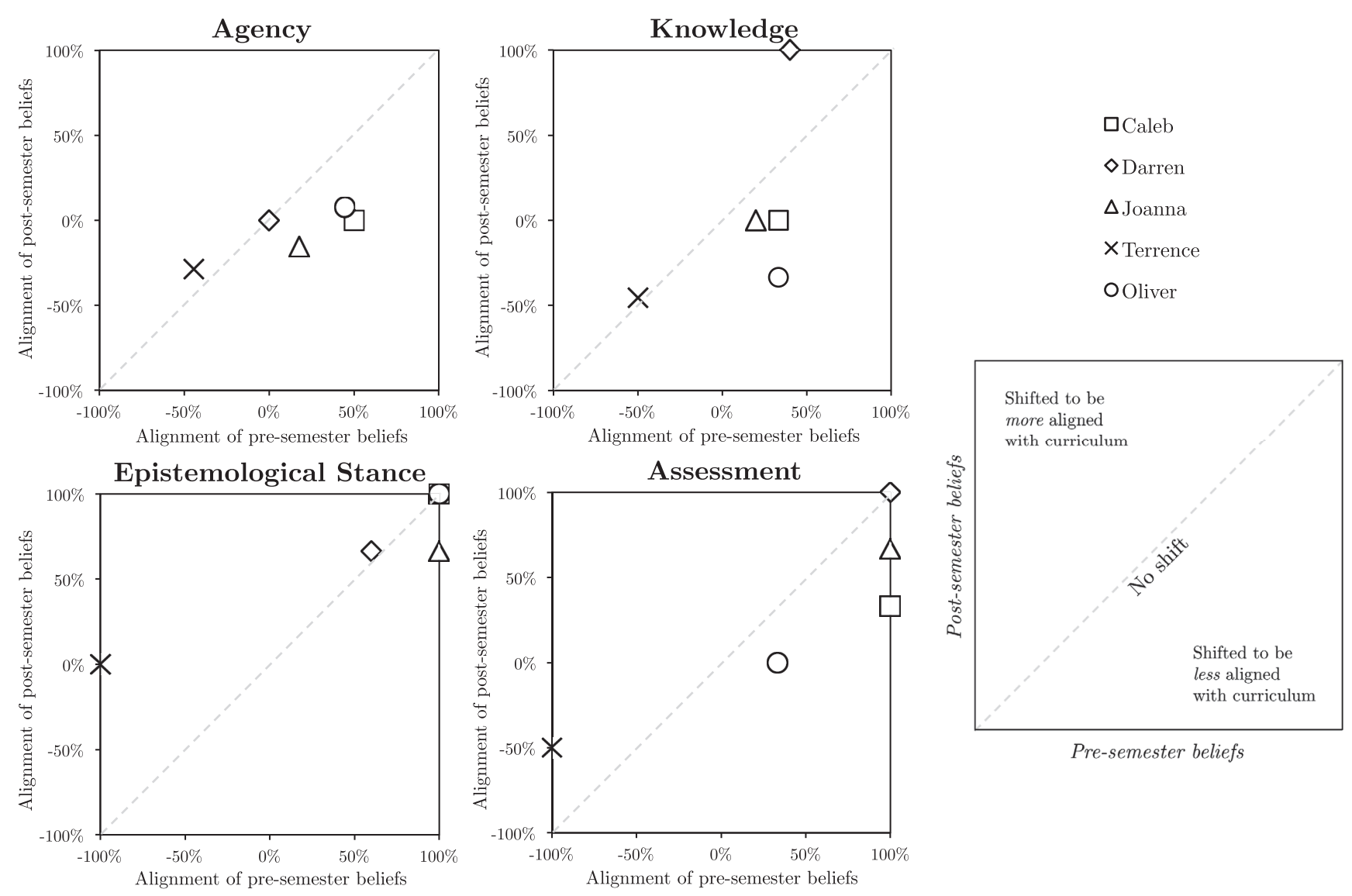

FIG. 8. Two-dimensional representation of pre-post semester shifts in TA beliefs. For each coding dimension, the marker position indicates the degree of alignment between the curriculum and pre-semester beliefs (horizontal) or post-semester beliefs (vertical). In this representation, off-diagonal points represent shifts. (See guide at right.) The three pilot TAs are not included, as pre-semester interviews were not conducted with them.

did not appreciably change their beliefs over the course of the semester. Terrence appeared to undergo large shifts along epistemological stance and assessment, but these may be exaggerated due to the small number of overall codes compared to other dimensions. The TA who appeared to undergo the smallest shifts was Darren, who was also notably consistent in using a specific metaphor ("translating information") to describe his teaching during interviews.

Although it is not apparent from the aggregate practices shown in Fig. 6, we did observe preliminary indications of differences in TAs' observed practices across different tutorials. However, we did not observe an overall progression toward more or less alignment with the tutorial over time. Rather, the individual tutorials tended to support and/or constrain TA practices on a week-by-week basis, which warrants further investigation.

\section{DISCUSSION AND CONCLUSIONS}

We conclude by returning to the motivating questions presented in Sec. III:
(1) Can we design an instrument that is able to distinguish stated beliefs and practices of physics TAs?

We have constructed a framework for comparing TA beliefs and practices along three primary dimensions, and demonstrated its utility in distinguishing among TAs within each of these dimensions. Figure 6 showed how the full set of 8 TAs compared in their post-semester coded beliefs along the TA-PIVOT dimensions. Furthermore, we were able to replicate our initial observation from pilot data that some TAs tended to be more aligned with certain aspects of the tutorial approach than others in their stated beliefs.

(2) Do we observe instances of coordination and/or discoordination between between beliefs and practices?

The expanded study uncovered additional instances of discoordination between TAs' beliefs and practices. Furthermore, the degree of coordination between TAs' beliefs and practices tended to vary across the TA-PIVOT dimensions. For instance, most of the TAs were coordinated in their beliefs and practices regarding knowledge goals, but not their epistemological stance. Some TAs, like Darren, exhibited a more consistent level of discoordination across dimensions. 
As we described previously, perceived barriers to implementation are one explanation for why instructors' observed practices may not coordinate with their stated beliefs. Talking to these instructors and presenting them with examples of their own practices affords a rich opportunity to approach the question of why they chose to engage in certain practices but not others. At the end of the post-semester interview during the expanded study, the five TAs commented on segments of video drawn from the video data inventory. In this case the TA video reflections provided additional information regarding the underpinning motives for these differences in approach. A number of preparation models include video consultations to scaffold practitioners' ability to reflect upon instructional practices, and we envision the TA-PIVOT framework serving to enhance these efforts (for instance, by allowing TAs to self-code).

(3) Can we observe shifts in TAs' beliefs over the course of a semester?

We were able to distinguish shifts in stated beliefs for the TAs as measured by the TA-PIVOT framework, although the size of the shifts were small compared to their overall span of coded statements. Hence it appears that TAs tended to persist in particular beliefs, despite teaching in a research-based classroom environment that is structured to promote student-centered instruction. In other words, it does not appear sufficient to simply place TAs in instructional environments that are aligned with student-centered pedagogy in order to impact their beliefs about teaching.

This result is consistent with prior research on the development of teacher beliefs, which suggests that preservice teachers' beliefs change over a time span of several years. Furthermore, four of the five TAs in the expanded study population entered with some form of teaching experience, some in environments very similar to the $\mathrm{CU}$ tutorial model. It is possible that these prior experiences served to engage these TAs in refining beliefs about teaching before their arrival at $\mathrm{CU}$, which may have reduced the "novel" impact of teaching in a transformed environment.

Although TAs' practices did not appear to undergo consistent overall shifts over the course of the semester, in analyzing the data we observed indications of week-byweek differences, suggesting that certain TAs tended to utilize different strategies for different tutorials. We hypothesize that the structure and/or content of the tutorials may be a factor in these differences in approach, and the TAPIVOT framework will be useful in future work examining the subtle parameters that influence TAs' choice of strategy.

In conclusion, we have developed a framework for the analysis of instructor beliefs and practices along three dimensions, which is grounded in both existing theory and emergent themes in the data drawn from interviews and observations of TAs. This TA-PIVOT framework serves to connect how TAs talk about their own teaching to the ways that they engage students in the classroom. In the process of constructing and applying the TA-PIVOT framework, we have observed (i) variation among TAs in their beliefs and practices, (ii) apparent instances of inconsistency between TAs' beliefs and practices, and (iii) no significant shifts in particular beliefs over time. These observations provide an opportunity to examine TAs' awareness and perception of how their classroom practices relate to their beliefs, and provide us as researchers the opportunity to investigate the nature and sources of tension between beliefs and practices. For instance, we would like to have TAs self-code their own practices as both a novel way to further test the framework and a targeted reflective opportunity for themselves.

We have designed the TA-PIVOT framework to be an adaptable tool that can be applied to a wide array of classroom settings. Now that we have established a baseline for utility of the framework in one research context, future work will allow us to further refine the coding schema and explore the framework's potential application to examining and improving professional development for TAs.

\section{APPENDIX A: INTERVIEW PROTOCOL}

Presemester only:

- Do you have any prior teaching experience?

- What were your undergraduate physics recitations like? What were your physics TAs like?

- How would you describe how you learn best?

- Why did you decide to go into physics?

Mid- or postsemester only:

- How are things going? or How did the semester go?

- What is teaching, to you?

- As you see it right now, how would you describe your role as a TA?

- If you had complete freedom to create the ideal environment for learning physics, what would it look like?

- What do you think students should learn in a firstsemester physics course? Do you think everyone should learn physics?

- Based on what you've seen of the tutorials, what about them do you think is good for students? What about them would you change?

- How do you think group work is helpful for students? Do you think it's helpful for all students?

- Walk me through one of your sections.

- How do you decide to start interacting with a group of students?

- When you do, how does it start?

- How do you know when to leave a group and move on?

- At the beginning of the section, does the TA need to give any sort of introduction to the tutorial, or just have students get started?

- Is there any experience this semester that stands out to you? 


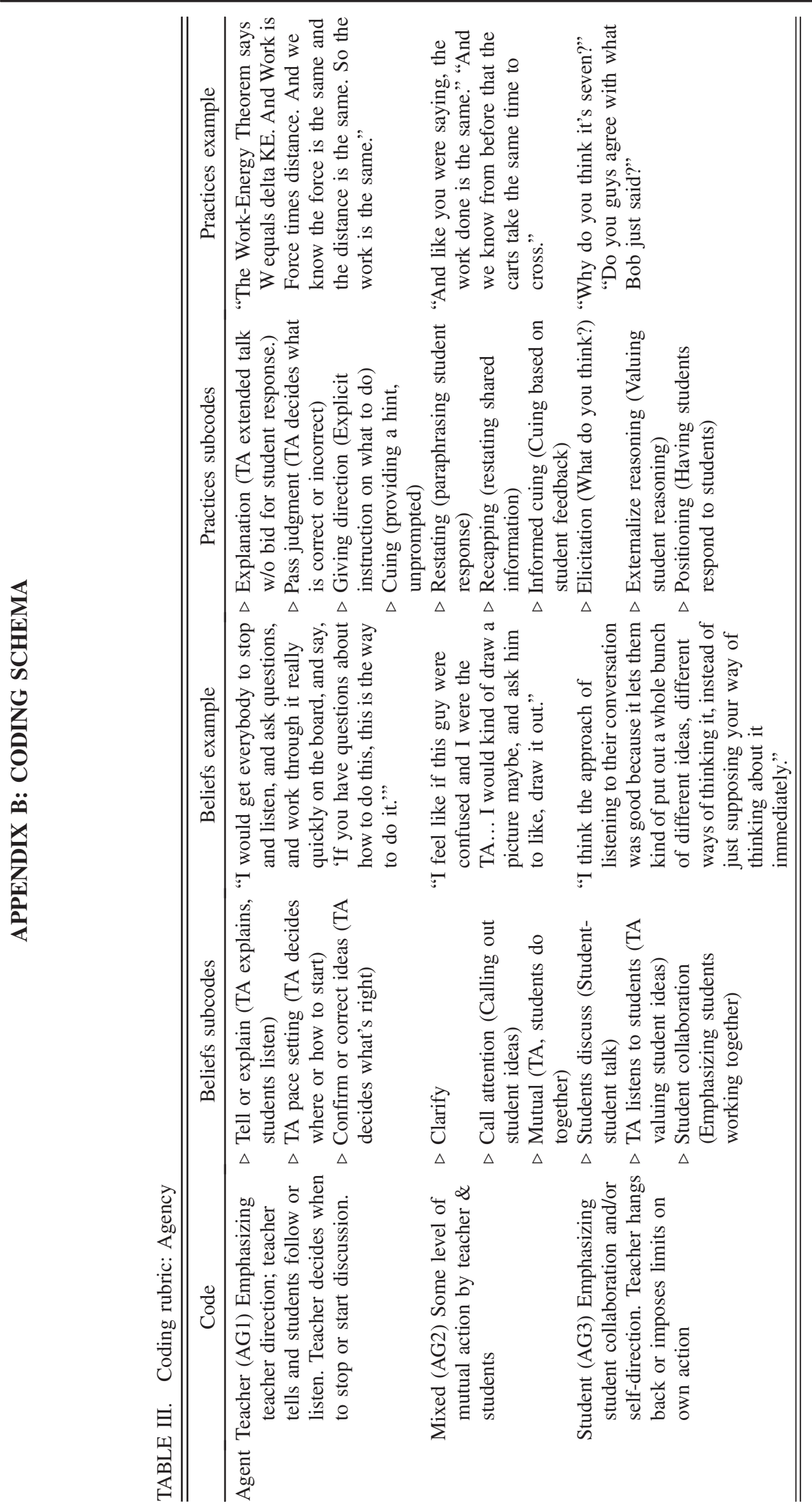




\begin{tabular}{|c|c|c|c|c|c|c|}
\hline & 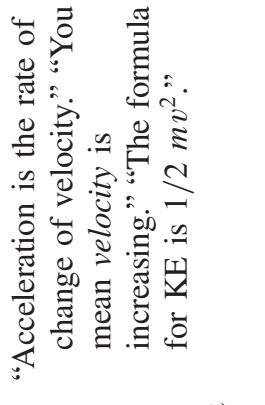 & 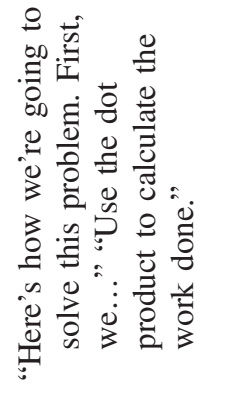 & 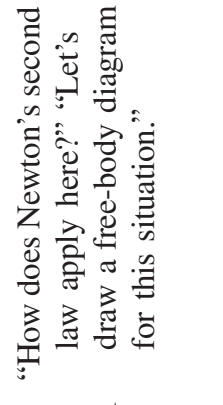 & 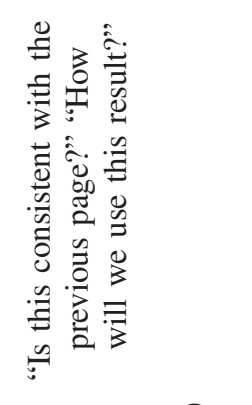 & 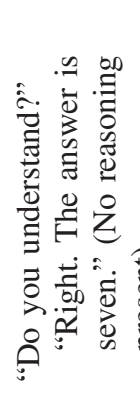 & 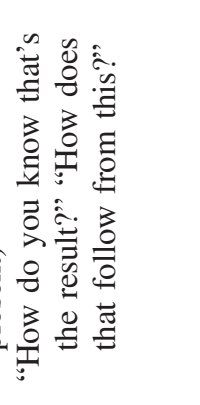 \\
\hline & 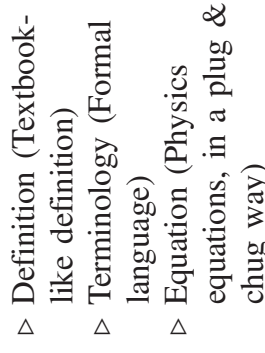 & 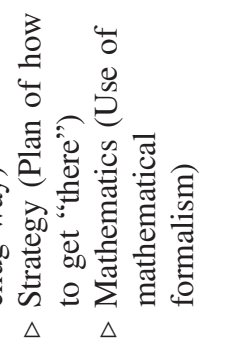 & 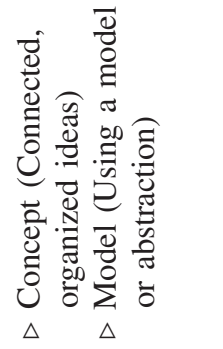 & 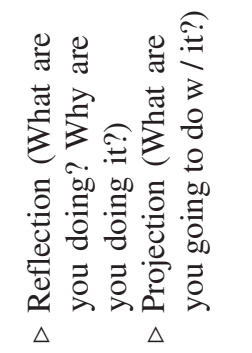 & 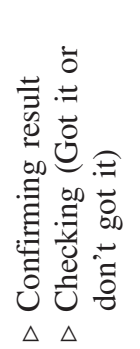 & 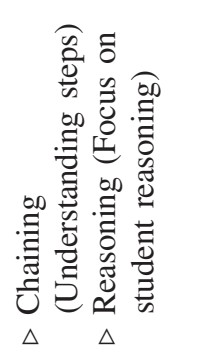 \\
\hline & 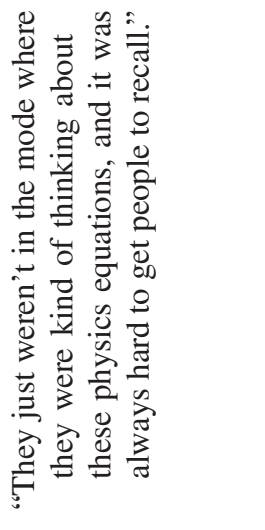 & 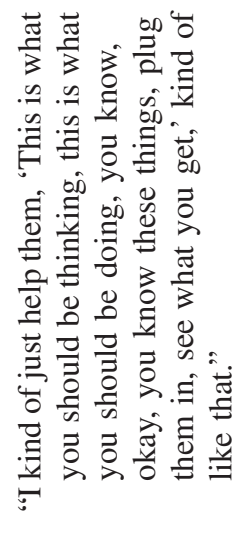 & 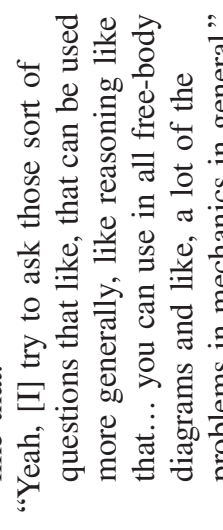 & 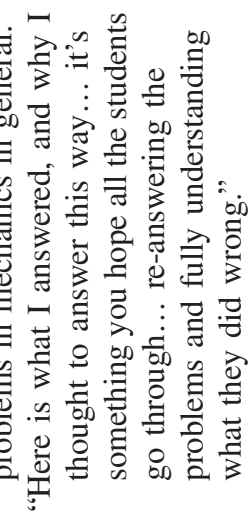 & 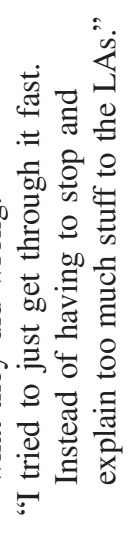 & 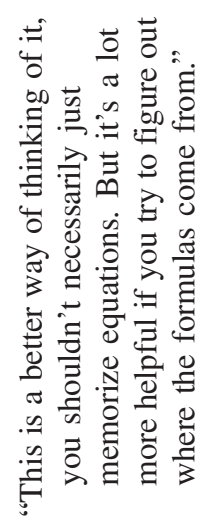 \\
\hline & 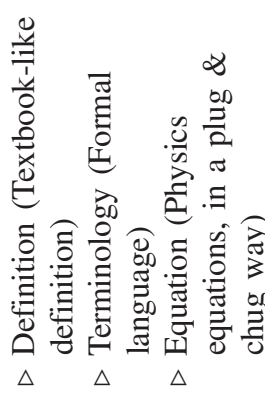 & 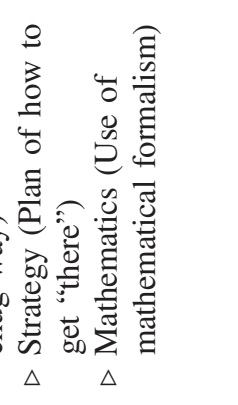 & 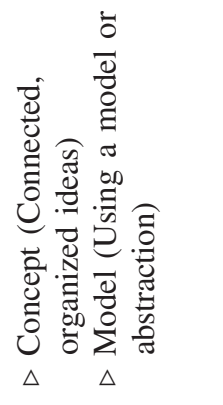 & 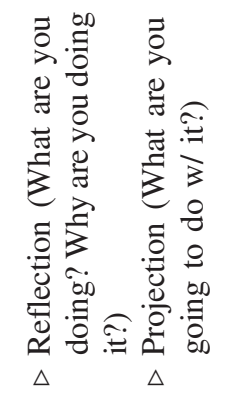 & 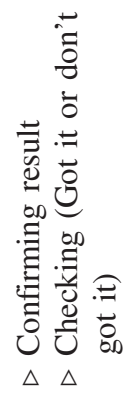 & 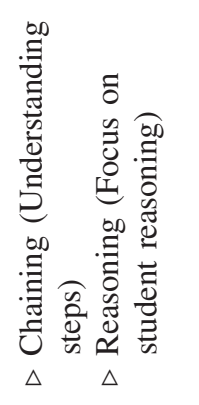 \\
\hline $\begin{array}{l}8 \\
\end{array}$ & 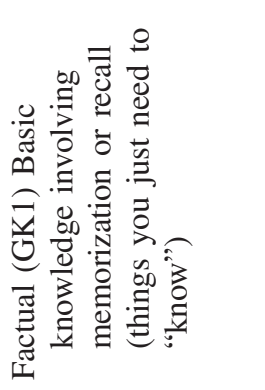 & 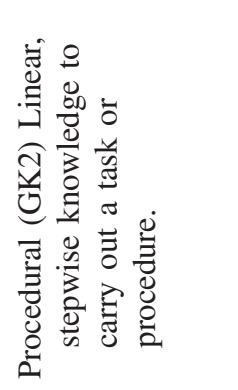 & 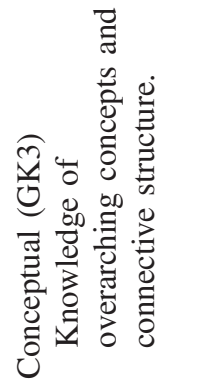 & 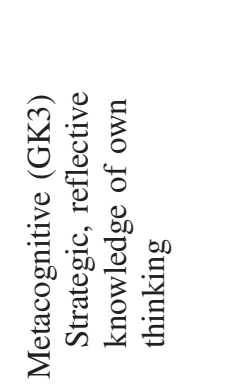 & 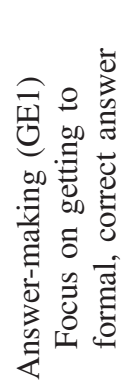 & 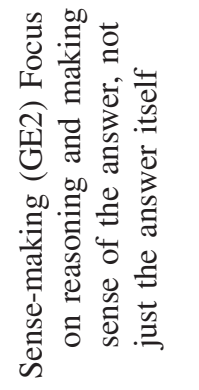 \\
\hline & & & & & 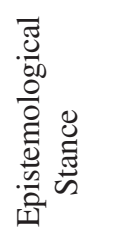 & \\
\hline
\end{tabular}




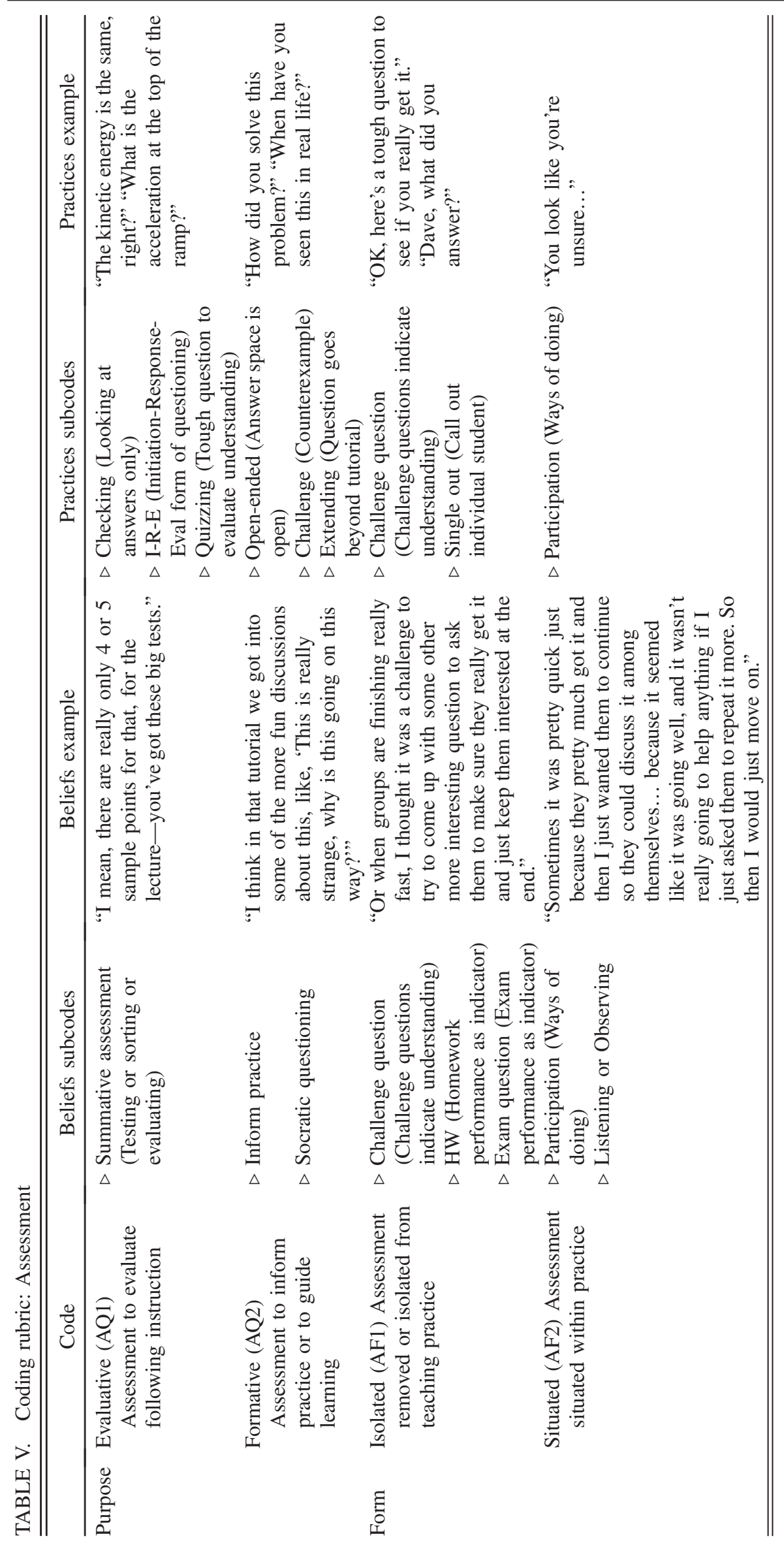


[1] National Academies, Rising Above the Gathering Storm: Energizing and Employing America for a Brighter Economic Future (National Academies Press, Washington, DC, 2005).

[2] S. Olson and D. G. Riordan, "Engage to excel: Producing one million additional college graduates with degrees in science, technology, engineering, and mathematics. report to the president", Executive Office of the President (2012).

[3] J. Handelsman, D. Ebert-May, R. Beichner, P. Bruns, A. Chang, R. DeHaan, J. Gentile, S. Lauffer, J. Stewart, and S. M. Tilghman, Scientific teaching, Science 304, 521 (2004).

[4] R. R. Hake, Interactive-engagement versus traditional methods: A six-thousand-student survey of mechanics test data for introductory physics courses, Am. J. Phys. 66, 64 (1998).

[5] C. Turpen and N. Finkelstein, The construction of different classroom norms during peer instruction: students perceive differences, Phys. Rev. ST Phys. Educ. Res. 6, 020123 (2010).

[6] L. C. McDermott and P.S. Shaffer, Tutorials in Introductory Physics 1st ed. (Prentice Hall, Englewood Cliffs, NJ, 2002).

[7] R. M. Goertzen, R. E. Scherr, and A. Elby, Indicators of understanding: what TAs listen for in student responses, in Proceedings of the 2008 Physics Education Research Conference, edited by C. Henderson, M. Sabella, and L. Hsu (2008), pp. 119-122.

[8] R. M. Goertzen, R. E. Scherr, and A. Elby, Accounting for tutorial teaching assistants' buy-in to reform instruction, Phys. Rev. ST Phys. Educ. Res. 5, 020109 (2009).

[9] K. M. Koenig and R. J. Endorf, Study of TA's ability to implement the Tutorials in Introductory Physics and student conceptual understanding, in Proceedings of the 2003 Physics Education Research Conference, edited by J. Marx, S. Franklin, and K. Cummings (2004), pp. 161-164.

[10] K. M. Koenig, R. J. Endorf, and G. A. Braun, Effectiveness of different tutorial recitation teaching methods and its implications for TA training, Phys. Rev. ST Phys. Educ. Res. 3, 010104 (2007).

[11] A. E. Austin, M. R. Connolly, and C. L. Colbeck, Strategies for preparing integrated faculty: The center for the integration of research, teaching, and learning, New Directions for Teaching and Learning 113, 69 (2008).

[12] B. T. Spike and N. D. Finkelstein, Toward an analytic framework of physics teaching assistants' pedagogical knowledge, in Proceedings of the 2011 Physics Education Research Conference, edited by N.S. Rebello, P. V Engelhardt, and C. Singh (2012), pp. 363-366.

[13] B. T. Spike and N. D. Finkelstein, Applying a framework for characterizing physics teaching assistants' beliefs and practices, in Proceedings of the 2012 Physics Education Research Conference, edited by P. V Engelhardt, A. D Churukian, and N. S. Rebello (2013), pp. 394-397.

[14] M. Pajares, Teachers' beliefs and educational research: Cleaning up a messy construct, Rev. Educ. Res. 62, 307 (1992).

[15] D. B. McLeod and S. H. McLeod, Synthesis-beliefs and mathematics education: implications for learning, teaching, and research, in Beliefs: A Hidden Variable in Mathematics
Education? (Kluwer Academic, Dordrecht, 2003), pp. $115-123$.

[16] A. G. Thompson, Teachers' beliefs and conceptions: A synthesis of the research, in Handbook of Research on Mathematics Teaching and Learning, edited by D. A. Grouws (Macmillan Publishing Co, Inc., New York, 1992), pp. 127-146.

[17] J. Nespor, The role of beliefs in the practice of teaching, J. Curric. Stud. 19, 317 (1987).

[18] D. J. Trumbull, Evolving conceptions of teaching: Reflections of one teacher, Curriculum Inquiry 20, 161 (1990).

[19] J. Aguirre and N. Speer, Examining the relationship between beliefs and goals in teacher practice, J. Math. Behav. 18, 327 (1999).

[20] M. Cole, Cultural Psychology, A Once and Future Discipline (Harvard University, Cambridge, MA, 1996).

[21] N. Finkelstein, Learning physics in context: A study of student learning about electricity and magnetism, Int. J. Sci. Educ. 27, 1187 (2005).

[22] C. Hoyles, Mathematics teaching and mathematics teachers: A meta-case study, For the Learning of Mathematics 12, 32 (1992).

[23] A. G. Thompson, The relationship of teachers' conceptions of mathematics and mathematics teaching to instructional practice, Educ. Stud. Math. 15, 105 (1984).

[24] R. M. Goertzen, R. E. Scherr, and A. Elby, Tutorial teaching assistants in the classroom: Similar teaching behaviors are supported by varied beliefs about teaching and learning, Phys. Rev. ST Phys. Educ. Res. 6, 010105 (2010).

[25] N. M. Speer, Connecting beliefs and practices: A finegrained analysis of a college mathematics teacher's collections of beliefs and their relationship to his instructional practices, Cognit. Instr. 26, 218 (2008).

[26] R. Kane, S. Sandretto, and C. Heath, Telling half the story: A critical review of research on the teaching beliefs and practices of university academics, Rev. Educ. Res. 72, 177 (2002).

[27] M. Piburn, D. Sawada, J. Turley, K. Falconer, R. Benford, I. Bloom, and E. Judson, Reformed teaching observation protocol (RTOP) reference manual, Tempe, Arizona: Arizona Collaborative for Excellence in the Preparation of Teachers (2000).

[28] M. T. Hora, A. Oleson, and J. J. Ferrare, Teaching dimensions observation protocol (TDOP) user's manual, tdop .wceruw.org (2013).

[29] M. K. Smith, F. H. M. Jones, S. L. Gilbert, and C. E. Wieman, The classroom observation protocol for undergraduate stem (COPUS): a new instrument to characterize university stem classroom practices, Cell Biol. Educ. 12, 618 (2013).

[30] E. R. Singer, Espoused teaching paradigms of college faculty, Res. High. Educ. 37, 659 (1996).

[31] M. Prosser, E. Martin, K. Trigwell, P. Ramsden, and H. Middleton, University academics' experience of research and its relationship to their experience of teaching, Instr. Sci. 36, 3 (2008).

[32] J. Haney and J. McArthur, Four case studies of prospective science teachers' beliefs concerning constructivist teaching practices, Sci. Educ. 86, 783 (2002). 
[33] Technical Report of the 2008 Graduate Education Conference (2009).

[34] C. Henderson and M. H. Dancy, Barriers to the use of research-based instructional strategies: The influence of both individual and situational characteristics, Phys. Rev. ST Phys. Educ. Res. 3, 020102 (2007).

[35] R. E. Scherr, R. S. Russ, T. J. Bing, and R. A. Hodges, Initiation of student-TA interactions in tutorials, Phys. Rev. ST Phys. Educ. Res. 2, 020108 (2006).

[36] N. D. Finkelstein and S. J. Pollock, Replicating and understanding successful innovations: Implementing tutorials in introductory physics, Phys. Rev. ST Phys. Educ. Res. 1, 010101 (2005).

[37] R. J. Beichner et al., Introduction to scale-up: Studentcentered activities for large enrollment university physics, in Proceedings of the 2000 Annual meeting of the American Society for Engineering Education (2000).

[38] V. Otero, N. Finkelstein, R. McCray, and S. Pollock, Who is responsible for preparing science teachers?, Science $\mathbf{3 1 3}$, 445 (2006).

[39] V. K. Otero and D. B. Harlow, "Getting started in Qualitative Physics Education Research", In Reviews in PER Vol. 2: Getting Started in Physics Education Research (2009).

[40] L. M. Ahearn, Language and agency, Annual review of anthropology 30, 109 (2001).

[41] E. Mazur, Peer Instruction: A User's Manual, Prentice Hall Series in Educational Innovation (Prentice Hall, Englewood Cliffs, NJ, 1997).
[42] M. Dancy and C. Henderson, Framework for articulating instructional practices and conceptions, Phys. Rev. ST Phys. Educ. Res. 3, 010103 (2007).

[43] L. W. Anderson, D. R. Krathwohl, and B. S. Bloom, A Taxonomy for Learning, Teaching, and Assessing (Longman, New York, 2001).

[44] E. Kashy, B. M. Sherrill, Y. Tsai, D. Thaler, D. Weinshank, M. Engelmann, and D. J. Morrissey, CAPA-An integrated computer-assisted personalized assignment system, Am. J. Phys. 61, 1124 (1993).

[45] T. M. Kuhs and D. L. Ball, Approaches to teaching mathematics: Mapping the domains of knowledge, skills, and dispositions, East Lansing: Michigan State University, Center on Teacher Education (1986).

[46] L. C. McDermott, Millikan Lecture 1990: What we teach and what is learned-Closing the gap, Am. J. Phys. 59, 301 (1991).

[47] H. Mehan, Learning Lessons: Social Organization in the Classroom (Harvard University, Cambridge, MA, 1979).

[48] C. Turpen, M. Dancy, and C. Henderson, Faculty perspectives on using peer instruction: A national study, in Proceedings of the 2010 Physics Education Research Conference, edited by C. Singh, N. S. Rebello, and M. Sabella (2010), pp. 325-328.

[49] C. Henderson and M. H. Dancy, Am. J. Phys. 76, 79 (2008). 